\title{
1 The divergent restoration effects of Lactobacillus strains in
}

\section{2 antibiotic-induced dysbiosis}

3 Ying Shi ${ }^{\text {a,b,c,e }}$, Lee Kellingray ${ }^{\text {d,e }}$, Gwenaelle Le Gall ${ }^{\text {e }}$, Jianxin Zhao ${ }^{\text {a }}$, Hao Zhang ${ }^{\text {a,b }}$, Arjan

4 Narbad $^{\mathrm{d}, \mathrm{e}^{*}}$, Qixiao Zhai ${ }^{\mathrm{a}, \mathrm{b}, \mathrm{c}^{*}}$, Wei Chen ${ }^{\mathrm{a}, \mathrm{b}, \mathrm{f}}$

5

6 a State Key Laboratory of Food Science and Technology, Jiangnan University, Wuxi, Jiangsu

$7 \quad 214122$, China

8 b National Engineering Research Centre for Functional Food, Wuxi, Jiangsu 214122, China

9 c International Joint Research Laboratory for Probiotics at Jiangnan University, Wuxi, Jiangsu

10 214122, China

11 d UK-China Joint Centre on Probiotic Bacteria, Norwich, NR4 7UA, United Kingdom

12 e Gut Microbes and Health Programme, Quadram Institute Bioscience, Norwich, NR4 7UA,

13 United Kingdom

14 f Beijing Innovation Centre of Food Nutrition and Human Health, Beijing Technology and

15 Business University (BTBU), Beijing 100048, China

*Co-corresponding authors:

18 Qixiao Zhai

19 Address: State Key Laboratory of Food Science and Technology, Jiangnan University, Wuxi, 20 Jiangsu 214122, China.

21 E-mail: zhaiqixiao@sina.com, Phone: (86)510-85912155 
23 Arjan Narbad

24 Quadram Institute Bioscience, Norwich, NR4 7UA, United Kingdom.

25 E-mail: arjan.narbad@quadram.ac.uk, Phone: (44)1603 255000 


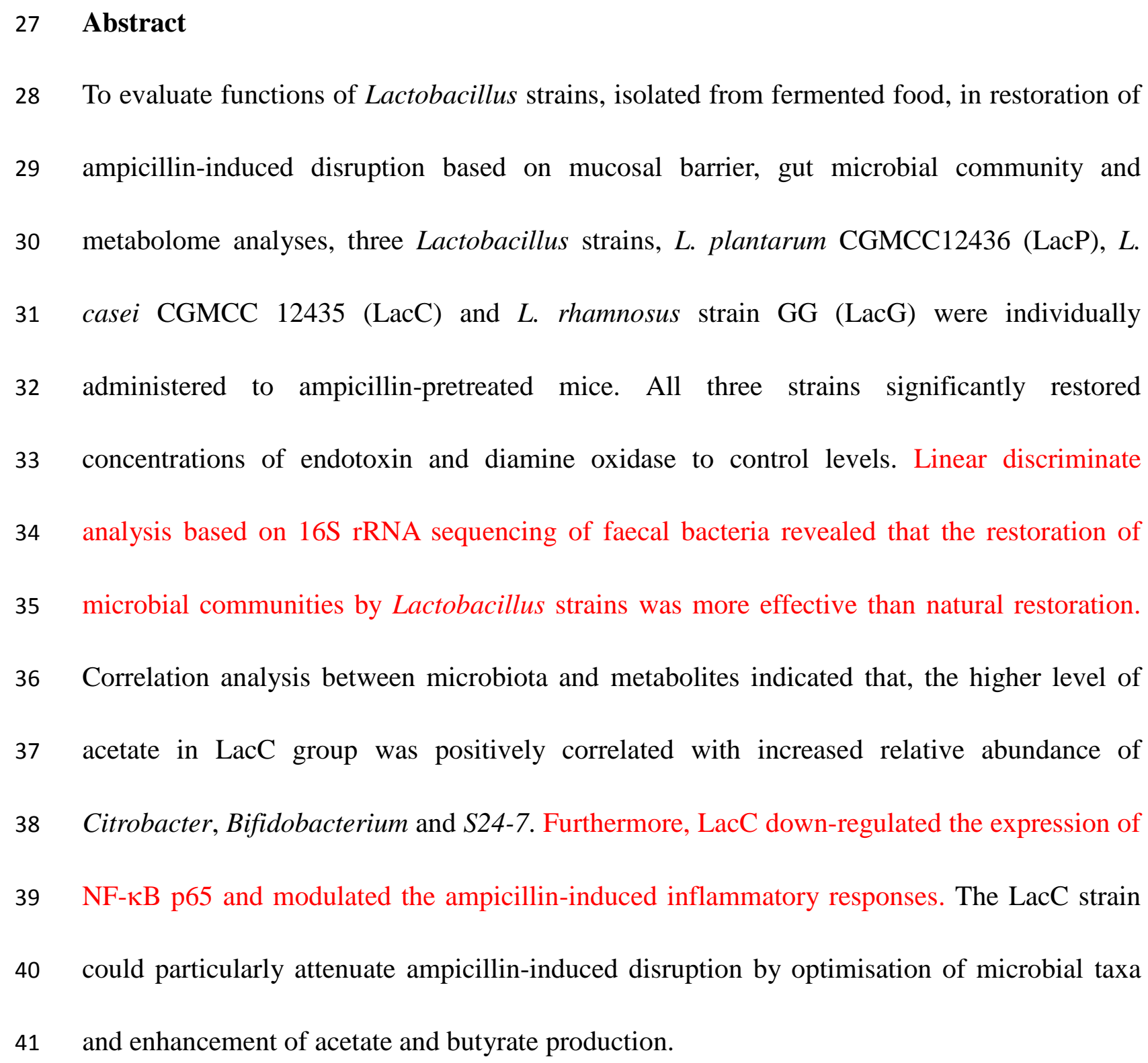




\section{Introduction}

The intestinal distal gastrointestinal tract is colonised by trillions of microbes, and this extensive microbial community comprising approximately $10^{12} \mathrm{CFU}$ in the colon, influences gastrointestinal physiology, metabolism, immunity and susceptibility to disease infection (Lozupone, Stombaugh, Gordon, Jansson, \& Knight, 2012). The disruption of the "balanced" microbiota, referred to as dysbiosis, can drive functional and inflammatory changes in animals and humans (Petersen \& Round, 2014). In the last decade, a large number of studies have announced significant alterations in the structure of microbial communities in patients and mice models of dysbiosis-related diseases such as inflammatory bowel diseases, diabetes, obesity, asthma and autism (Becattini, Taur, \& Pamer, 2016; Karlsson et al., 2013). Alterations to the intestinal microbiota during a critical developmental period also had lasting metabolic consequences (Abrahamsson et al., 2014; Parracho, Bingham, Gibson, \& McCartney, 2005). Antibiotic-induced dysbiosis was linked to changes in colonic microbial ecology; ampicillin has been shown to decrease the number of Bifidobacteria, increase Candida, and reduce the production of short-chain fatty acids (SCFA) (Hawrelak \& Myers, 2004; Mangin, Suau, Gotteland, Brunser, \& Pochart, 2010). It was suggested that, due to the inability to differentiate between commensals and pathogens, antibiotics perturbed the microbiota structure and the evolutionary relationship between the immune system and the host symbionts (Aguilera, Cerda-Cuellar, \& Martinez, 2015; Buffie et al., 2012; Cho et al., 2012).

Antibiotic use could induce dysregulation of metabolic activities conducted by colonic microbiota (Lee \& Hase, 2014). Microbial metabolites are capable of manipulating the metabolic integrity of intestinal epithelial cells and causing intestinal immune responses 
(Arpaia \& Rudensky, 2014). Some bacterial fermentation products, particularly SCFA, were considered as key signs of colonic health, but the specific relationship between the microbial community and metabolites under the status of antibiotic-induced dysbiosis is poorly understood. The intestinal immune response should be balanced between the tolerance for unexpected immune molecules and pathogen-induced inflammation in the host cells and commensal bacteria, with the balance developed by mucus production and antimicrobial peptides to establish a barrier between host tissue and the microbes (Johansson, Larsson, \& Hansson, 2011; Vaishnava et al., 2011). Toll-like receptors (TLRs) are a group of pattern recognition receptors, that play a crucial role in mucosal immune response (de Kivit, Tobin, Forsyth, Keshavarzian, \& Landay, 2014) and can recognise microbe-associated molecular patterns (MAMPs). As one of these MAMPs, lipopolysaccharide (LPS) can be increased through antibiotic use and cause an innate immune response via TLR4 in intestinal epithelial cells (Collado-Romero, Arce, Ramirez-Boo, Carvajal, \& Garrido, 2010). It is well accepted that LPS initiates a signalling pathway through TLR4 to activate NF- $\mathrm{BB}$, and leads to inflammation and removal of infection by pro-inflammatory cytokines including TNF- $\alpha$ and IL-1 $\beta$ (Doyle \& O'Neill, 2006). One strain of Lactobacillus has been proven to down-regulate the expression of pro-inflammatory cytokines in a TLR4-dependent NF- $\mathrm{B}$ signal pathway (Shimazu et al., 2012).

A number of Lactobacillus strains, tested as cocktails or individual strains, were shown to alleviate gut-related disorders or metabolic diseases such as obesity, diabetes and non-alcoholic fatty liver disease in mice (Aronsson et al., 2010; Simon et al., 2015; Wang et al., 2015; Xu, Wan, Fang, Lu, \& Cai, 2012; Yoo et al., 2013). Importantly, different probiotic 
strains were proven to have remarkably different abilities to modulate gut metabolism and immune response (Fåk \& Bäckhed, 2012; Million et al., 2012; Yin, Yu, Fu, Liu, \& Lu, 2010).

It remains unclear whether specific Lactobacillus strains can be administered to regulate the alteration of the gut microbiota and subsequently promote the production of beneficial metabolites. Moreover, it has been reported that a Lactobacillus mixture played a beneficial role in the immune response in mice through balancing anti- or pro-inflammatory cytokines (Taranu, Marin, Pistol, Motiu, \& Pelinescu, 2015).

In the present study, a model of dysbiosis was constructed by exposing healthy adult mice to a therapeutic-dose of ampicillin, leading to perturbed gut microbiota. The ampicillin-induced dysbiosis was confirmed by determining the caecal index, endotoxin levels and the expression of tight-junction proteins. The different Lactobacillus species ( $L$. plantarum, L. casei and L. rhamnosus) were compared to investigate their impact on the restoration of microbiota in an ampicillin-induced dysbiotic state. The metabolic composition of faecal water was measured using ${ }^{1} \mathrm{H}$ NMR, and the relationship between the microbial community and SCFA was correlated to characterise the function of Lactobacillus strains tested. Our findings provide a novel insight that the administration of different Lactobacillus strains after ampicillin-induced dysbiosis exhibited distinct effects in modulation of the microbial community, metabolites and the immune system.

\section{Materials and Methods}

2.1 Culturing of bacteria

L. plantarum CGMCC12436 and L. casei CGMCC12435 were isolated from a traditional 
111 fermented cream from Inner Mongolia, China, and the L. rhamnosus strain GG (LGG) (ATCC 112 533103) was purchased from ATCC. All strains were held in long-term storage $\left(-80^{\circ} \mathrm{C}\right.$ in $30 \%$ 113 sterile glycerol) in the Culture Collection of the Food Microbiology (CCFM) of Jiangnan 114 University. These three strains were cultured in de Man Rogosa and Sharpe (MRS) broth at

$37^{\circ} \mathrm{C}$ overnight. The bacterial cultures were centrifuged and the pellets were resuspended in

$0.9 \%$ saline solution to give a final concentration of $\sim 1 \times 10^{9} \mathrm{CFU}$ per $0.2 \mathrm{~mL}$ respectively.

\subsection{Experimental animals and ethics statement}

The experiments were carried out with four-week-old male C57BL/6 mice obtained from Slack Experimental Animal Co., LTD (Shanghai, China). Mice were caged in groups of two or three. Throughout the experiments, distilled water and standard laboratory chow were provided ad libitum. Light conditions (12 h light/dark cycle), temperature $\left(21^{\circ} \mathrm{C}\right)$ and air humidity were tightly controlled. The experimental procedures and numbers of animals used were approved by the Ethics Committee of Jiangnan University in China (JN No. 20160608-20160831/47). The experiments were designed in order that both the number of animals used and their suffering were minimised.

\subsection{Experimental groups, timelines and treatment}

Mice were allocated to one of six groups (Table 1) and acclimatised for 1 week. Ampicillin (Sigma, USA) was dissolved in $0.9 \%$ saline solution (500 $\mathrm{mg} \mathrm{kg}^{-1}$ ), and mice were treated via oral gavage with ampicillin for 2 weeks, except for the control group (Con, $\mathrm{n}=20$-24) which were treated with $0.9 \%$ saline solution for 2 weeks. After 2 weeks, the group of dysbiotic mice (Amp) and half the group of control mice were sacrificed, whilst the remaining control mice received saline via oral gavage for another 4 weeks; the natural 
restoration group (NaR) pre-treated with ampicillin received saline via oral gavage for 4 weeks; dysbiotic mice were treated via oral gavage with $L$. rhamnosus GG (LacG group), $L$. plantarum CGMCC12436 (LacP group), or L. casei CGMCC12435 (LacC group) respectively, for 4 weeks.

2.4 Quantification of gut permeability in the serum

The concentrations of endotoxin (ET) and diamine oxidase (DAO) were determined in serum samples using Enzyme-Linked Immunosorbent Assay (ELISA) kits (SenBeiJia Biological Technology Co. Ltd., Nanjing, China). Gut permeability was also measured using 4000 Da fluorescent dextran-FITC (DX-4000-FITC) (Sigma-Aldrich, USA) as described in the supplementary methods.

\subsection{Microbiome analysis}

Total genomic DNA was extracted from thawed faecal samples with the FastDNA Spin Kit for Soil (MP Biomedical, USA) according to the manufacturer's instructions. Subsequently, the 16S rRNA gene was amplified by PCR with a forward (5'- CCT AYG GGR BGC ASC AG -3') and reverse (5'- GGA CTA CNN GGG TAT CTA AT -3') barcoded primer 148 set, targeting the V3-V4 region. PCR products were gel-purified and the amplicon DNA 149 concentration was determined, the libraries were prepared using the TruSeq DNA LT Sample 150 Preparation Kit (Illumina, USA) and sequenced on the Illumina MiSeq platform (500 cycles 151 paired-end). The detailed methods of QIIME and Linear Discriminate Analysis (LDA) with 152 Effect Size (LEfSe) analyses are described in the supplementary methods.

$153 \quad 2.6$ Faecal metabolomic analysis

154 Metabolites in the faecal samples of mice were analysed by ${ }^{1} \mathrm{H}$ NMR analysis at 
Quadram Institute Bioscience (United Kingdom). Faecal water was prepared by mixing 100 mg of frozen faecal samples with 12 times the volume of a phosphate buffer that consisted of $1.9 \mathrm{mM} \mathrm{Na} \mathrm{HPO}_{4}, 8.1 \mathrm{mM} \mathrm{NaH} \mathrm{PO}_{4}, 150 \mathrm{mM} \mathrm{NaCl}$, and $1 \mathrm{mM}$ TSP (sodium 3-(trimethylsilyl)-propionate- $\left.\mathrm{d}_{4}\right)$ ) in $\mathrm{D}_{2} \mathrm{O}$ (deuterium oxide). After homogenising thoroughly

with a pellet pestle motor (Kimble Kontes, USA), samples were centrifuged at 14,000 $g$ for 10 min at $4^{\circ} \mathrm{C}$. High resolution ${ }^{1} \mathrm{H}$ NMR spectra were recorded using a Bruker AV 600 spectrometer (Bruker, Rheinstetten, Germany). The spectra were analysed as previously described and further plotting is listed in the supplementary methods (Le Gall et al., 2011).

\subsection{Correlation between taxa abundance and metabolites}

To evaluate the relationship between the most abundant taxa and observed metabolites, a correlation test was performed, and associated $p$-values were adjusted for multiple testing in $\mathrm{R}$. The physeq package was used to obtain taxa abundance and meta data information, the ggplot2 package was used to plot figures, "Pearson" was selected as the method to characterise correlation coefficient, and "adjust meta variables (panel on the correlation plot)" was selected to adjust $p$-values for multiple comparisons using Benjamin and Hochberg.

\subsection{ELISA analysis of inflammatory cytokines in the colon}

Colon tissues (100 mg) were homogenised in $900 \mu \mathrm{L}$ PBS using a Scientz-50 tissue mill (Lanzhi, Ningbo, China), centrifuged at $13,000 \mathrm{~g}$ for $10 \mathrm{~min}$ at $4^{\circ} \mathrm{C}$, and the supernatants were transferred into sterile tubes. The levels of secretory immunoglobulin A (sIgA), nuclear factor kappa-light-chain-enhancer of activated B cells (NF-қB), monocyte chemotactic protein 1 (MCP-1), interferon- $\gamma($ IFN- $\gamma)$, regenerating islet derived protein 3 gamma (Reg3 $\gamma)$ and interleukin $1 \beta$ ( IL-1 $\beta$ ) were determined using respective ELISA kits following the 
17 3

manufacturer's protocols (Nanjing SenBeiJia Biological Technology Co. Ltd. China).

\subsection{Immunofluorescence}

Colon tissue sections from different groups of mice were fixed by $4 \%$ paraformaldehyde in $0.1 \mathrm{M}$ phosphate buffer. The tissues were excised, post fixed for $3 \mathrm{~h}$ in the perfusion fixative, cryoprotected for $72 \mathrm{~h}$ in $30 \%$ sucrose in $0.1 \mathrm{M}$ phosphate buffer. Transverse sections $(20 \mu \mathrm{m})$ were cut and the slides were incubated with primary NF- $\kappa \mathrm{B}$ p65 rabbit polyclonal antibody (Thermo Fisher Scientific, USA) (1:200 dilution) for $2 \mathrm{~h}$ at $37^{\circ} \mathrm{C}$. Following incubation, sections were washed in PBS and incubated with secondary goat anti-rabbit antibody (1:100 in PBS) (Jackson Immuno Research, USA) for $1 \mathrm{~h}$ in a dark room. The sections were incubated with 4,6-Diamidino-2-phenylindole dihydrochlorid (DAPI) (Sigma Aldrich), washed twice, and visualised under a Leica fluorescence microscope. All micrographs were taken with identical exposure times and focused on the centre of each well.

\subsection{Statistical analysis}

Data were represented as mean \pm standard error of the mean (SEM). The gut permeability data were analysed using one-way ANOVA, followed by Dunnett's multiple comparisons test 192 in GraphPad Prism 5. The mRNA expression of tight-junction proteins, metabolites and levels 193 of cytokines were calculated using one-way ANOVA followed by Tukey's multiple 194 comparisons test in GraphPad Prism 5. $P<0.05$ was considered statistically significant.

1952.11 Data deposition

The raw sequence data have been deposited in the NCBI Sequence Read Archive 197 (Accession no. SRP146081 and BioProject Accession no. PRJNA471394). 


\section{Results}

3.1 Effects of Lactobacillus strains on intestinal injury in antibiotic-induced dysbiotic mice

Gut permeability and the caecal index were investigated as indicators of alteration to intestinal integrity. The Amp mice showed significantly increased levels of DX-4000-FITC in serum and caecal index, which indicated an increase in gut permeability and an enlargement of the caecum after antibiotic use compared to the Con group ( $p<0.0001$, Fig. $1 \mathrm{~A}$ and B). The endotoxin concentration was increased ( $p<0.0001$, Fig. 1C) and the enzyme activity of serum DAO was decreased in the Amp group compared to the Con group ( $p<0.01$, Fig. 1D), demonstrating the damage of mucosal integrity after antibiotic exposure. After four-weeks restoration, no difference was observed among these four intestinal integrity biomarkers in the natural restoration group (NaR) compared to controls. Treatment with LacC markedly reduced the level of DX-4000-FITC and caecal index, and proved to be more effective than LacG and LacP (Fig. 1A and B). In addition, all groups of Lactobacillus treatment modified the levels of endotoxin and DAO towards the control level (Fig. 1C and D).

3.2 Effects of Lactobacillus strains on intestinal barrier disruption in antibiotic-induced dysbiotic mice

To evaluate potential effects of Lactobacillus treatment on paracellular communications 216 in the intestines, we measured mRNA expression of the tight-junction proteins ZO-1, 217 Occludin and Claudin-1 in the colon and ileum (Fig. 2). In the colon, the expression of ZO-1 218 and Occludin were not statistically affected by ampicillin-induced dysbiosis, however, 219 expression of Claudin-1 was significantly decreased $(p<0.001)$ after antibiotic use. In the 220 ileum, levels of all tight-junction proteins were remarkably reduced by ampicillin use ( $p<$ 
$2210.01, p<0.0001$ and $p<0.001$ respectively). LacC treatment enhanced the expression of 222 ZO-1 and Occludin to the control level in the ileum which was more effective than LacG ( $p<$ 2230.05 and $p<0.001$ respectively), while LacP promoted the expression of Claudin- 1 in the 224 ileum to the control level. These data demonstrated that, with regards to the expression of 225 tight-junction proteins, the disruption by ampicillin and the enhancement by Lactobacillus 226 strains mainly occurred in the ileum, and the LacC strain showed a promotion of ZO-1 and 227 Occludin while the LacP strain positively affected Claudin-1 levels.

3.3 Ampicillin-induced colonic microbiome disruption can be largely restored by Lactobacillus administration

Microbial species richness was indicated by the inverse of the classical Simpson diversity (Invsimpson Index) as shown in Fig. 3A, which was calculated to eliminate the sampling effects of the Shannon Index. The bacterial diversity was greatly reduced by ampicillin treatment ( $p<0.001$ ), and three groups of the administration of single Lactobacillus strain enhanced the level of alpha-diversity to that of the Con group, which was higher than that observed in the NaR group ( $p<0.001$ ). Principal coordinate analysis (PCoA) based on Phyloseq's Weighted Unifrac showed that ampicillin-treated mice had a considerably altered (clustering by distance) microbial community compared to that of the Con mice (Fig. 3B and C). The profiles of the microbial composition of the Con group and Lactobacillus-restored groups were clustered more closely to each other than that of naturally-restored mice (Fig. 3B and C), indicating that tested Lactobacillus strains could restore the antibiotic-treated microbiota structure towards the normal profile.

Ampicillin treatment led to an increase in Proteobacteria and a severe depletion of 
Bacteroidetes and Verrucomicrobia at the phylum level (Fig. 3D). Natural restoration did not lead to the recovery of Bacteroidetes, while LacC, LacG and LacP groups had an increase in the abundance of Bacteroidetes towards, or above ( $\mathrm{LacC}$ ), that of the Con group. Although the microbiota was not completely restored, the abundance of Proteobacteria was improved to the control level by LacC and LacP strains, but not by the LacG strain.

We further examined compositional changes of the microbiome at the family or genus level using high-throughput amplicon sequencing. Following antibiotic cessation, taxa including Enterobacteriaceae, Klebsiella and Enterococcus were dramatically increased in Amp mice compared to the Con mice, whereas Akkermansia, Lachnospiraceae and Dorea were absent in the Amp group (Fig. S1). The cluster of the NaR grouped closer to the Amp group rather than the Con group, indicating that natural restoration for four weeks after ampicillin disruption was not effective in recovering the microbial community to the normal level (Fig. S2). An evaluation of relative abundance (Fig. S4) indicated that Coprobacillus, S24-7 and Eubacterium were enhanced in the LacC, LacG and LacP groups after ampicillin-induced depletion, whilst there was no observed restoration in the NaR group. Meanwhile, the relative abundance of Klebsiella and Enterococcus was reduced by LacC, LacG and LacP strains after their increase due to ampicillin exposure. These data indicated that each of the three Lactobacillus strains altered the community structure of the gut microbiota in a manner different from the ampicillin-induced dysbiotic state towards that of the Con group. Furthermore, the LEfSe analysis revealed that the number of significantly altered taxa was lower in LacC (15) than NaR (25) (Fig. 4A and B). The significantly altered taxa after administration of LacG or LacP was 15 and 13 respectively (Fig. S3), demonstrating 
that restoration of the microbiota by Lactobacillus strains enabled a stronger shift towards the

initial state than observed by natural restoration.

3.4 The LacC strain restored the faecal metabolome following alteration by ampicillin-induced dysbiosis

The impact of ampicillin-induced microbial community alterations on the faecal metabolite profiles was evaluated by ${ }^{1} \mathrm{H}$ NMR spectroscopy, which revealed that ampicillin exposure altered the faecal metabolome composition as can be seen by the clear separation between the Amp group and the Con group (Fig. 5A). The LacC group clustered closer to the Con group than the LacG or LacP groups, with NaR positioned between the Con and Amp groups, indicating that LacC was more effective in restoration of the faecal metabolome than natural recovery or LacG and LacP strains. Some metabolites, such as amino acids and carbohydrates produced by the colonic microbiota, were found to have decreased in the faecal samples of ampicillin-treated mice (Fig. S5). In particular, the SCFA reflected by acetate, propionate and butyrate were significantly decreased or diminished $(p<0.0001$ ) by the antibiotic use (Fig. 5B).

The administration of Lactobacillus strains could partly restore the SCFA profile, however, strain-dependent differences were observed. Acetate, propionate and butyrate levels were found to be significantly enhanced in the LacC group compared to the Amp group ( $p<$ 0.0001, Fig. 5B), increasing towards the same levels as seen in the Con group. The LacG group increased the levels of acetate and propionate to levels higher than the NaR group, but had less effect in the recovery of butyrate levels. However, SCFA levels were not significantly altered in the LacP group. In addition, the increases in lactate levels observed following 
3

ampicillin administration were significantly reduced by all three Lactobacillus groups and the NaR group ( $p<0.0001$, Fig. 5B). Taken together, these results showed that the LacC strain mitigated the decrease of SCFA in ampicillin-treated mice and was more effective in the restoring the whole metabolic profile.

3.5 The relationship between altered faecal metabolome and changes in the microbial community

To assess microbiota-metabolome associations, the functional correlation between alterations in microbial taxa and metabolites was assessed using Pearson's correlation coefficient method, based on 60 bacterial taxa and 8 acids that potentially contributed to the observed differences between the Amp and Con groups (Fig. 5C). Acetate, butyrate and succinate were highly correlated with alterations in proportions of bacterial taxa following ampicillin use. The decrease in acetate within the Amp group was positively correlated with the decreased relative abundance of Staphylococcus, Streptophyta and Planococcaceae ( $p<$ 0.01). The decreased level of butyrate was positively correlated with changes in the relative abundance of Rikenellaceae, Helicobacter, Lactobacillus, Lactobacillaceae, and Epulopiscium whilst was negatively correlated with Morganella, Enterobacter and Enterobacteriaceae in the Amp group. In particular, the enhanced level of acetate in the LacC group was positively correlated with an increase in the relative abundance of Citrobacter, Bifidobacterium, Eubacterium, S24-7, Rikenellaceae and Clostridiaceae ( $p<0.05$, Fig. 5D), and was negatively correlated with members of Ruminococcus, Ruminococcaceae, Dorea, Coprococcus, Bilophila, Lachnospiraceae and Desulfovibrionaceae ( $p<0.05$, Fig. 5D). 308 However, no significant correlations were observed between microbial taxa and butyrate in 
either the LacC group or LacP (Fig. 5D, E).

3.6 Lactobacillus strains modulated NF-кB signalling and colonic inflammation caused by ampicillin-induced dysbiosis

The serum endotoxin in ampicillin-treated mice was significantly increased $(p<0.0001$, Fig. 1C) compared to the Con mice, indicating that LPS-stimulated TLR4 and NF- $\mathrm{B}$ expression might be induced following ampicillin exposure. NF- $\kappa$ B levels determined by ELISA (Fig. 6B) were significantly increased $(p<0.0001)$ after ampicillin treatment, and reduced to control levels by the administration of LacC or LacP. Consistent with the ELISA

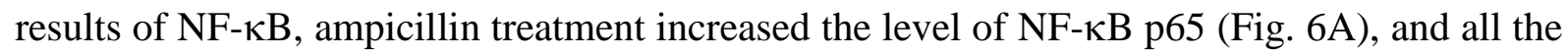
tested Lactobacillus strains could partly reduce the level of p65 compared to that achieved by

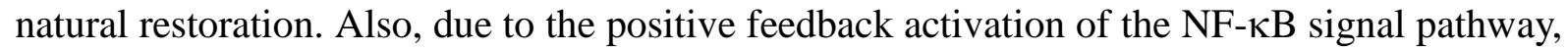
the level of IL-1 $\beta$ was significantly increased by ampicillin treatment ( $p<0.0001)$, and reduced by LacC to the level of the Con group (Fig. 6B). Therefore, the LacC strain seemed to protect against ampicillin-induced inflammatory responses through the regulation of NF-kB expression in the colon.

To investigate whether disturbance of the microbiota induced alterations in mouse 325 intestinal immune homeostasis, the local expression levels of several inflammatory mediators 326 were measured in the colon. In addition, the effects of different Lactobacillus strains against 327 inflammatory mediator expression were also evaluated. Levels of secretory IgA (sIgA) were 328 significantly increased in antibiotic-induced mice $(p<0.0001)$ whilst LacG and LacC reduced 329 the levels of sIgA to that of the Con group. In ampicillin-treated mice, the expression of 330 Reg3 $\gamma$ was increased in the colon, and decreased in the LacG group compared to the NaR 
group ( $p<0.0001$, Fig. 6B).

The inflammatory cytokines including IFN- $\gamma$ and MCP-1 were also examined to evaluate colonic inflammation. Levels of IFN- $\gamma$ and MCP-1 were considerably increased in the Amp group compared to the Con group ( $p<0.0001)$. IFN- $\gamma$ was modulated towards the level observed in the Con group by the LacC group but none of the Lactobacillus groups could reverse MCP-1 to the same level as the Con group. Taken together, inflammatory mediators were activated in the colon following treatment with ampicillin, and the LacC strain was more effective in modulating the levels of sIgA, Reg3 $\gamma$ and IFN- $\gamma$ whilst LacG administration reduced the level of Reg3 $\gamma$.

\section{Discussion}

By generating ampicillin-induced dysbiosis in the gut microbiota and related metabolome, we could analyse the chronic effects of ampicillin on the host and investigate restoration strategies (Scott et al., 2016). Previous studies applied mouse models to explore the relationship between antibiotic treatment and subsequent changes in host physiology and gut 346 microbiota composition (Aguilera et al., 2015; Bech-Nielsen et al., 2012; Mahana et al., 2016; 347 van Opstal et al., 2016). Although some informative alterations can be measured by 348 examining the microbiota or the metabolome alone, correlation analyses were employed in 349 this study to assess the relationship between the microbiota and metabolome and provide 350 functional information. Furthermore, by comparing the effects of the three Lactobacillus 351 strains tested here, the present study revealed substantial evidence of associations between 352 microbial taxa and metabolites in the ampicillin-induced and Lactobacillus-restored mice. 
The Amp mice exhibited a decreased microbial diversity. A reduction in microbial diversity is associated with multiple gastrointestinal diseases (Guarner, 2015; Le Chatelier et al., 2013). Several microbial taxa including Enterobacteriaceae, Clostridia, Erwinia, and Klebsiella were found to be enhanced in the NaR mice (after ampicillin treatment). Among these changed taxa, the high frequency of Klebsiella and Enterobacteriaceae has been observed in patients with gastroenteritis and irritable bowel syndrome (Ganji et al., 2016). In the Amp mice, the increased relative abundance of Enterobacteriaceae and decreased Lachnospiraceae were strongly associated with gut inflammation. The reduction of Klebsiella and Enterococcus and the enhancement of Coprobacillus, Bacteroidales and Eubacterium in all three Lactobacillus treatment groups suggested that Lactobacillus administration contributed to the promotion of a stable gut microbial community. In particular, the relative abundance of S24-7 (family) was enhanced in the LacC group and these butyrate-producing bacteria are beneficial to intestinal epithelial cell health (Villanueva-Millan, Perez-Matute, \& Oteo, 2015). The decrease of the SCFA was not only associated with perturbation of the microbial communities but also related to the integrity of mucosal barrier in the Amp group. SCFA are reported to be associated with maintenance of intestinal barrier function; acetate was shown to be crucial in the inhibition of enteropathogens (Fukuda et al., 2011), and 370 butyrate production could lead to increased mucin production and promotion of tight-junction 371 integrity (Jung, Park, Jeon, \& Han, 2015). The majority of gut butyrate-producers including 372 Faecalibacterium, Eubacterium, and Roseburia utilise pathway in which butyryl-CoA is 373 converted to butyrate (Louis, Young, Holtrop, \& Flint, 2010). In this study, following an 374 initial decrease due to ampicillin exposure, the abundance of Eubacterium improved in each 
of the Lactobacillus administration groups, which may explain the increase of butyrate observed in these groups.

The correlation between microbial communities and faecal metabolites provided crucial evidence on the function of bacteria, with alterations at the microbiota-level leading to a changed metabolome, which could potentially influence gut disease (Claesson et al., 2012; Tremaroli \& Bäckhed, 2012). We observed a positive correlation between the relative abundance of S24-7 (belong to Bacteroidales) and levels of faecal acetate and butyrate. It was previously reported that consumption of common bean and chickpea reduced colitis-associated inflammation, whilst promoting the levels of SCFA and S24-7 (Power et al., 2016). After the administration of the LacC strain, acetate levels were significantly enhanced, and this increase was positively correlated with the relative abundance of Citrobacter, Bifidobacterium, Eubacterium and Rikenellaceae in this study. Among these acetate-related gut bacteria, the selective increase of Bifidobacterium has been shown to protect against enteropathogenic infection through the enhancement of acetate (Fukuda et al., 2011); in an elderly health study, genera including Eubacterium were associated with long-stay subjects and acetate production, as well as gene counts for acetate-producing enzymes, were significantly higher in long-stay subjects (Claesson et al., 2012). Interestingly, the increased abundance of Rikenellaceae, which was associated with the increase of acetate in the LacC group, had previously been associated with potentially beneficial effects on gut health. The decrease in Rikenellaceae observed in the Amp and NaR groups had recently been observed in the intestinal dysbiosis of spondyloarthritis (Lin et al., 2014). These analyses of the 396 functional relationship between microbial taxa and SCFA further specified the possible 
mechanism of functional restoration of LacC strain in the metabolome and microbiome.

Exposure to ampicillin increases levels of endotoxin, activation of the NF- $\mathrm{kB}$ pathway and upregulation of the pro-inflammatory cytokines, which are in accordance with the observations during LPS exposure (Lawrence, 2009). The SCFA-driven inhibition of histone deacetylases (HDACs) tends to improve an anti-inflammatory cell phenotype that is critical for maintaining immune homeostasis. A number of studies identified the inactivation of NF-kB and downregulation of pro-inflammatory cytokines by SCFA (Usami et al., 2008; Vinolo et al., 2011). Also, LPS-induced expression of pro-inflammatory cytokines was attenuated by Lactobacillus jensenii through the down-regulation of the TLR4-dependent NF-kB pathway and the mitogen-activated protein kinase (MAPK) in a porcine intestinal epithelial cell line (Shimazu et al., 2012). In this study, L. casei (LacC), L. plantarum (LacP) and $L$. rhamnosus GG (LacG) were administrated to ampicillin-treated mice and the levels of NF-kB and IL-1 $\beta$ were found to be reduced in the colon by LacC. Collectively, these results confirm that LacC treatment could modulate the host immune responses through the TLR4-dependent NF-אB pathway in ampicillin-induced mice, although as yet it is unclear whether this was through the action of SCFA or a beneficial modulation of the microbiome structure.

\section{Conclusions}

416 We demonstrated that the three strains of different Lactobacillus species are able to 417 individually restore antibiotic-induced alterations of the microbiome and the metabolic profile 418 in mice. We found significant differential changes in colonic microbial taxa and clades by 

3

LEfSe analysis through the comparison of three Lactobacillus-restored groups with a natural restoration group. Correlation analysis of associations between the microbiota and metabolome indicated that LacC strain can promote specific bacterial taxa and SCFA to attenuate ampicillin-induced dysbiosis, suggesting strain-specific effects on functionally relevant gut disease. Furthermore, we confirmed that LacC reduced inflammatory activity by

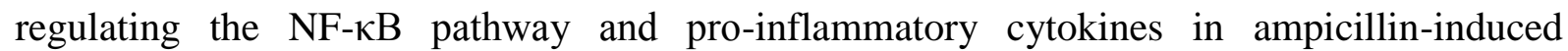
dysbiotic mice. These strain-specific Lactobacillus treatments offer the potential to mediate antibiotic-associated gastrointestinal disturbances and diseases, although clinical trials would be necessary to confirm their potential beneficial effects in humans.

\section{Acknowledgements}

This work was supported by the National Natural Science Foundation of China Key 431 Program (No. 31820103010), National First-Class Discipline Program of Food Science and 432 Technology (JUFSTR20180102), BBSRC Newton Fund Joint Centre Award (BB/J004529/1), 433 the National Natural Science Foundation of China (Grant 31470161), National Natural 434 Science Foundation of Jiangsu Province (BK20160175), National Natural Science Foundation 435 of China (No. 31601452) and Collaborative innovation center of food safety and quality 436 control in Jiangsu Province.

$439 \quad$ All authors declared no conflict of interest. 


\section{References}

Abrahamsson, T. R., Jakobsson, H. E., Andersson, A. F., Bjorksten, B., Engstrand, L., \& Jenmalm, M. C. (2014). Low gut microbiota diversity in early infancy precedes asthma at school age. Clinical and Experimental Allergy, 44(6), 842-850. doi: 10.1111/cea.12253

Aguilera, M., Cerda-Cuellar, M., \& Martinez, V. (2015). Antibiotic-induced dysbiosis alters host-bacterial interactions and leads to colonic sensory and motor changes in mice. Gut Microbes, 6(1), 10-23. doi: 10.4161/19490976.2014.990790

Aronsson, L., Huang, Y., Parini, P., Korach-Andre, M., Hakansson, J., Gustafsson, J. A., ... Rafter, J. (2010). Decreased fat storage by Lactobacillus paracasei is associated with increased levels of angiopoietin-like 4 protein (ANGPTL4). Plos One, 5(9). doi: ARTN e1308710.1371/journal.pone.0013087

Arpaia, N., \& Rudensky, A. Y. (2014). Microbial metabolites control gut inflammatory responses. Proceedings of the National Academy of Sciences of the United States of America, 111(6), 2058-2059. doi: 10.1073/pnas.1323183111

Becattini, S., Taur, Y., \& Pamer, E. G. (2016). Antibiotic-induced changes in the intestinal microbiota and disease. Trends in Molecular Medicine, 22(6), 458-478. doi: 10.1016/j.molmed.2016.04.003

Bech-Nielsen, G. V., Hansen, C. H. F., Hufeldt, M. R., Nielsen, D. S., Aasted, B., Vogensen, F. K., ... Hansen, A. K. (2012). Manipulation of the gut microbiota in C57BL/6 mice changes glucose tolerance without affecting weight development and gut mucosal immunity. Research in Veterinary Science, 92(3), 501-508. doi: 10.1016/j.rvsc.2011.04.005

Buffie, C. G., Jarchum, I., Equinda, M., Lipuma, L., Gobourne, A., Viale, A., ... Pamer, E. G. (2012). Profound alterations of intestinal microbiota following a single dose of clindamycin results in sustained susceptibility to Clostridium difficile-induced colitis. Infection and Immunity, 80(1), 62-73. doi: 10.1128/Iai.05496-11

Cho, I., Yamanishi, S., Cox, L., Methé, B. A., Zavadil, J., Li, K., ... Blaser, M. J. (2012). Antibiotics in early life alter the murine colonic microbiome and adiposity. Nature, 
488(7413), 621-626. doi: 10.1038/nature11400

Claesson, M. J., Jeffery, I. B., Conde, S., Power, S. E., O'connor, E. M., Cusack, S., ... O'sullivan, O. (2012). Gut microbiota composition correlates with diet and health in the elderly. Nature, 488(7410), 178-184. doi: 10.1038/nature11319.

Collado-Romero, M., Arce, C., Ramirez-Boo, M., Carvajal, A., \& Garrido, J. J. (2010). Quantitative analysis of the immune response upon Salmonella typhimurium infection along the porcine intestinal gut. Veterinary Research, 41(2). doi: Artn 2310.1051/Vetres/2009072

de Kivit, S., Tobin, M. C., Forsyth, C. B., Keshavarzian, A., \& Landay, A. L. (2014). Regulation of intestinal immune responses through TLR activation: implications for pro- and prebiotics. Frontiers in Immunology, 5. doi: Artn 6010.3389/Fimmu.2014.00060

Doyle, S. L., \& O'Neill, L. A. J. (2006). Toll-like receptors: from the discovery of NF-kB to new insights into transcriptional regulations in innate immunity. Biochemical Pharmacology, 72(9), 1102-1113. doi: 10.1016/j.bcp.2006.07.010

Fåk, F., \& Bäckhed, F. (2012). Lactobacillus reuteri prevents diet-induced obesity, but not atherosclerosis, in a strain dependent fashion in Apoe-/- mice. PloS one, 7(10), e46837.

Fukuda, S., Toh, H., Hase, K., Oshima, K., Nakanishi, Y., Yoshimura, K., ... Ohno, H. (2011). Bifidobacteria can protect from enteropathogenic infection through production of acetate. Nature, 469(7331), 543-571. doi: 10.1038/nature09646

Ganji, L., Alebouyeh, M., Shirazi, M. H., Eshraghi, S. S., Mirshafiey, A., Ebrahimi Daryani, N., \& Zali, M. R. (2016). Dysbiosis of fecal microbiota and high frequency of Citrobacter, Klebsiella spp., and Actinomycetes in patients with irritable bowel syndrome and gastroenteritis. Gastroenterology and Hepatologyy from Bed to Bench, 9(4), 325-330.

Guarner, F. (2015). The gut microbiome: What do we know? Clinical Liver Disease, 5(4), 86-90. doi: doi:10.1002/cld.454

Hawrelak, J. A., \& Myers, S. P. (2004). The causes of intestinal dysbiosis: a review. 
Alternative Medicine Review, 9(2), 180-197.

Johansson, M. E. V., Larsson, J. M. H., \& Hansson, G. C. (2011). The two mucus layers of colon are organized by the MUC2 mucin, whereas the outer layer is a legislator of host-microbial interactions. Proceedings of the National Academy of Sciences of the United States of America, 108, 4659-4665. doi: 10.1073/pnas.1006451107

Jung, T. H., Park, J. H., Jeon, W. M., \& Han, K. S. (2015). Butyrate modulates bacterial adherence on LS174T human colorectal cells by stimulating mucin secretion and MAPK signaling pathway. Nutrition Research and Practice, 9(4), 343-349. doi: 10.4162/nrp.2015.9.4.343

Karlsson, F. H., Tremaroli, V., Nookaew, I., Bergstrom, G., Behre, C. J., Fagerberg, B., ... Backhed, F. (2013). Gut metagenome in European women with normal, impaired and diabetic glucose control. Nature, 498(7452), 99-110. doi: 10.1038/nature12198

Lawrence, T. (2009). The nuclear factor NF-кB pathway in inflammation. Cold Spring Harbor Perspectives in Biology, 1(6), 1-11. doi: 10.1101/cshperspect.a001651

Le Chatelier, E., Nielsen, T., Qin, J. J., Prifti, E., Hildebrand, F., Falony, G., ... Consortium, M. (2013). Richness of human gut microbiome correlates with metabolic markers. Nature, 500(7464), 541-557. doi: 10.1038/nature12506

Le Gall, G., Noor, S. O., Ridgway, K., Scovell, L., Jamieson, C., Johnson, I. T., ... Narbad, A. (2011). Metabolomics of fecal extracts detects altered metabolic activity of gut microbiota in ulcerative colitis and irritable bowel syndrome. Journal of Proteome Research, 10(9), 4208-4218. doi: 10.1021/pr2003598

Lee, W. J., \& Hase, K. (2014). Gut microbiota-generated metabolites in animal health and disease. Nature Chemical Biology, 10(6), 416-424. doi: 10.1038/nchembio.1535

Lin, P., Bach, M., Asquith, M., Lee, A. Y., Akileswaran, L., Stauffer, P., ... Rosenbaum, J. T. (2014). HLA-B27 and human beta 2-microglobulin affect the gut microbiota of transgenic rats. Plos One, 9(8). doi: ARTN e10568410.1371/journal.pone.0105684

Louis, P., Young, P., Holtrop, G., \& Flint, H. J. (2010). Diversity of human colonic butyrate-producing bacteria revealed by analysis of the butyryl-CoA:acetate CoA-transferase gene. Environmental Microbiology, 12(2), 304-314. doi: 
10.1111/j.1462-2920.2009.02066.x

Lozupone, C. A., Stombaugh, J. I., Gordon, J. I., Jansson, J. K., \& Knight, R. (2012). Diversity, stability and resilience of the human gut microbiota. Nature, 489(7415), 220-230. doi: 10.1038/nature11550

Mahana, D., Trent, C. M., Kurtz, Z. D., Bokulich, N. A., Battaglia, T., Chung, J., ... Blaser, M. J. (2016). Antibiotic perturbation of the murine gut microbiome enhances the adiposity, insulin resistance, and liver disease associated with high-fat diet. Genome Medicine, 8. doi: ARTN 4810.1186/s13073-016-0297-9

Mangin, I., Suau, A., Gotteland, M., Brunser, O., \& Pochart, P. (2010). Amoxicillin treatment modifies the composition of Bifidobacterium species in infant intestinal microbiota. Anaerobe, 16(4), 433-438. doi: 10.1016/j.anaerobe.2010.06.005

Million, M., Angelakis, E., Paul, M., Armougom, F., Leibovici, L., \& Raoult, D. (2012). Comparative meta-analysis of the effect of Lactobacillus species on weight gain in humans and animals. Microbial Pathogenesis, 53(2), 100-108. doi: 10.1016/j.micpath.2012.05.007

Parracho, H. M. R. T., Bingham, M. O., Gibson, G. R., \& McCartney, A. L. (2005). Differences between the gut microflora of children with autistic spectrum disorders and that of healthy children. Journal of Medical Microbiology, 54(10), 987-991. doi: 10.1099/jmm.0.46101-0

Petersen, C., \& Round, J. L. (2014). Defining dysbiosis and its influence on host immunity and disease. Cellular Microbiology, 16(7), 1024-1033. doi: 10.1111/cmi.12308

Power, K. A., Monk, J. M., Lepp, D., Robinson, L. E., Wu, W. Q., Carey, C., ... McGillis, L. (2016). Common bean and chickpea supplemented diets beneficially enhance the colonic microenvironment and reduce colitis-associated inflammation. Faseb Journal, 30.

Scott, F. I., Horton, D. B., Mamtani, R., Haynes, K., Goldberg, D. S., Lee, D. Y., \& Lewis, J. D. (2016). Administration of antibiotics to children before age 2 years increases risk for childhood obesity. Gastroenterology, 151(1), 120-137. doi: 10.1053/j.gastro.2016.03.006 
Shimazu, T., Villena, J., Tohno, M., Fujie, H., Hosoya, S., Shimosato, T., ... Kitazawa, H. (2012). Immunobiotic Lactobacillus jensenii elicits anti-inflammatory activity in porcine intestinal epithelial cells by modulating negative regulators of the toll-like receptor signaling pathway. Infection and Immunity, 80(1), 276-288. doi: 10.1128/Iai.05729-11

Simon, M. C., Strassburger, K., Nowotny, B., Kolb, H., Nowotny, P., Burkart, V., ... Roden, M. (2015). Intake of Lactobacillus reuteri improves incretin and insulin secretion in glucose-tolerant humans: A proof of concept. Diabetes Care, 38(10), 1827-1834. doi: $10.2337 / \mathrm{dc} 14-2690$

Taranu, I., Marin, D. E., Pistol, G. C., Motiu, M., \& Pelinescu, D. (2015). Induction of pro-inflammatory gene expression by Escherichia coli and mycotoxin zearalenone contamination and protection by a Lactobacillus mixture in porcine IPEC-1 cells. Toxicon, 97, 53-63. doi: 10.1016/j.toxicon.2015.01.016

Tremaroli, V., \& Bäckhed, F. (2012). Functional interactions between the gut microbiota and host metabolism. Nature, 489(7415), 242-249. doi: 10.1038/nature11552.

Usami, M., Kishimoto, K., Ohata, A., Miyoshi, M., Aoyama, M., Fueda, Y., \& Kotani, J. (2008). Butyrate and trichostatin A attenuate nuclear factor kappa B activation and tumor necrosis factor alpha secretion and increase prostaglandin E-2 secretion in human peripheral blood mononuclear cells. Nutrition Research, 28(5), 321-328. doi: 10.1016/j.nutres.2008.02.012

Vaishnava, S., Yamamoto, M., Severson, K. M., Ruhn, K. A., Yu, X. F., Koren, O., ... Hooper, L. V. (2011). The antibacterial lectin RegIII gamma promotes the spatial segregation of microbiota and host in the intestine. Science, 334(6053), 255-258. doi: 10.1126/science.1209791

van Opstal, E., Kolling, G. L., Mooreii, J. H., Coquery, C. M., Wade, N. S., Loo, W. M., ... Warren, C. A. (2016). Vancomycin treatment alters humoral immunity and intestinal microbiota in an aged mouse model of Clostridium difficile infection. Journal of Infectious Diseases, 214(1), 130-139. doi: 10.1093/infdis/jiw071

Villanueva-Millan, M. J., Perez-Matute, P., \& Oteo, J. A. (2015). Gut microbiota: a key player 
in health and disease. A review focused on obesity. Journal of Physiology and Biochemistry, 71(3), 509-525. doi: 10.1007/s13105-015-0390-3

Vinolo, M. A. R., Rodrigues, H. G., Hatanaka, E., Sato, F. T., Sampaio, S. C., \& Curi, R. (2011). Suppressive effect of short-chain fatty acids on production of proinflammatory mediators by neutrophils. Journal of Nutritional Biochemistry, 22(9), 849-855. doi: 10.1016/j.jnutbio.2010.07.009

Wang, J. J., Tang, H., Zhang, C. H., Zhao, Y. F., Derrien, M., Rocher, E., ... Shen, J. (2015). Modulation of gut microbiota during probiotic-mediated attenuation of metabolic syndrome in high fat diet-fed mice. Isme Journal, 9(1), 1-15. doi: 10.1038/ismej.2014.99

Xu, R. Y., Wan, Y. P., Fang, Q. Y., Lu, W., \& Cai, W. (2012). Supplementation with probiotics modifies gut flora and attenuates liver fat accumulation in rat nonalcoholic fatty liver disease model. Journal of Clinical Biochemistry and Nutrition, 50(1), 72-77. doi: 10.3164/jcbn.11-38

Yin, Y. N., Yu, Q. F., Fu, N. A., Liu, X. W., \& Lu, F. G. (2010). Effects of four Bifidobacteria on obesity in high-fat diet induced rats. World Journal of Gastroenterology, 16(27), 3394-3401. doi: 10.3748/wjg.v16.i27.3394

Yoo, S. R., Kim, Y. J., Park, D. Y., Jung, U. J., Jeon, S. M., Ahn, Y. T., ... Choi, M. S. (2013). Probiotics L. plantarum and L. curvatus in combination alter hepatic lipid metabolism and suppress diet-induced obesity. Obesity, 21(12), 2571-2578. doi: 10.1002/oby.20428 

3

\section{Figure Legends}

Fig. 1. Effects of Lactobacillus strains administration after antibiotic disturbance on gut injury. A. Level of DX-4000-FITC measured by ELISA in serum samples from different mice groups. B. Caecal indexes weighted and calculated through caecum tissues from different groups. C. Endotoxin and D. DAO concentrations measured by ELISA in serum samples from all mice groups.

Mice of the Ampicillin group (Amp) were treated with ampicillin for 2 weeks, mice of the control group (Con) were treated with saline for 2 weeks or 6 weeks; the natural restoration group (NaR) pre-treated with ampicillin (2 weeks) received saline for another 4 weeks, and dysbiotic mice, through pre-treatment with ampicillin for 2 weeks were treated with either Lactobacillus rhamnosus GG (LacG group), L. plantarum CGMCC12436 (LacP group), or L. casei CGMCC12435 (LacC group) respectively, for 4 weeks. These group names were used throughout this research paper. One-way ANOVA followed by Dunnett's multiple comparisons test was used to determine statistical significance, and the $p$ value was obtained by comparing the mean of each group with the mean of the Con group, ns means no significant difference, ${ }^{*} p<0.05,{ }^{* *} p<0.01$, ${ }^{* * *} p<0.001,{ }^{* * * *} p<0.0001$. Values are represented as mean \pm SEM of 5-8 mice per group.

Fig. 2. The alleviation of intestinal barrier disruption by Lactobacillus treatment.

Real-time PCR analysis of ZO-1, Occludin and Claudin-1 mRNA expression normalised to $\beta$-actin in the colons and ileums of mice from Con, Amp, NaR, LacG, LacC and LacP groups. One-way ANOVA followed by Tukey's multiple comparisons test was used to determine statistical significance, and the $p$ value was obtained by comparing the mean of each group 
with the mean of the Con group, ns means no significant difference, ${ }^{*} p<0.05$, ${ }^{* *} p<0.01$, $* * * p<0.001$. Values are represented as mean \pm SEM of 5-8 mice per group.

Fig. 3. The restorative effect of Lactobacillus strains following ampicillin-induced dysbiosis via microbial diversity and bacterial abundance measurements.

A. Microbial $\alpha$-diversity of faecal samples indicated by Shannon Index, Simpson Index and Invsimpson Index. The richness of each sample was estimated in a phyloseq data object, and one-way ANOVA followed by Dunnett's multiple comparisons test was used to determine statistical significance, with the $p$ value obtained by comparing the mean of each group with the mean of the Con group, ns means no significant difference, ${ }^{*} p<0.05$, ${ }^{* *} p<0.01$, ${ }^{* * *} p<$ 0.001 .

B. Principal coordinates analysis (PCoA) and C. clustering of distance based on Phyloseq's Weighted Unifrac to present differences in microbial community structure between samples

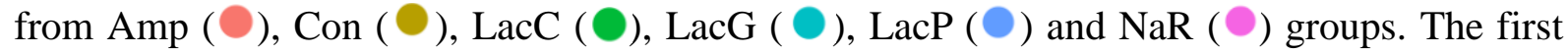
principal component (PC1) and second principal component (PC2) explained 51.6\% and 16.2\% of the variance in the Weighted UniFrac metrics, respectively. Each point represents the faecal microbiome of a single sample.

D. The abundance of bacterial phyla in faecal samples from Amp, Con, LacC, LacG, LacP and NaR mice groups. The rectangles representing Bacteroidetes, Firmicutes, Proteobacteria, Tenericutes and Verrucomicrobia were stacked in order and the aggregate height of the stacked bar reflects the quantitative information.

Fig. 4. Comparison of differential microbial communities from mice groups using LEfSe.

A. Circular cladogram of biologically and statistically consistent differences in faecal 

3

microbial clades between $\mathrm{NaR}$ and Con groups. In the panel, each circle's diameter was proportional to the taxon's abundance, green $=$ taxon significantly enriched in $\mathrm{NaR}$, red $=$ taxon significantly enriched in Con and yellow $=$ non-significant. The cladogram simultaneously highlights specific genera/families and high-level trends.

B. Histogram of LDA scores computed for taxa that have differential abundance in NaR and Con groups of mice. The magnitude of the LEfSe scores represents the degree of difference in relative abundance between features in the $\mathrm{NaR}$ and Con groups.

C. Circular cladogram of biologically and statistically consistent differences in faecal microbial clades between LacC and Con groups. Green = taxon significantly enriched in LacC, red $=$ taxon significantly enriched in Con and yellow $=$ non-significant. The cladogram simultaneously highlights specific genera/families and high-level trends.

D. Histogram of LDA scores computed for taxa that have differential abundance in LacC and Con groups of mice. The magnitude of the LEfSe scores represents the degree of consistent difference in relative abundance between features in the LacC and Con groups.

Fig. 5. The restoration effect of selected Lactobacillus strains after ampicillin-induced dysbiosis based on metabolomic analyses and the correlation between taxa abundance and metabolite alterations.

A. Principal component plot analysis of faecal metabolites altered in the Amp (๑), Con ( $\bullet)$, LacC (๑), LacG (๑), LacP (๑) and NaR (๑) groups. The first principal component (PC1) and second principal component (PC2) explained 36.8\% and $17.6 \%$ of the variance respectively. Each point represents the faecal metabolites in a single sample.

B. Distribution of intensities for the selected four acids based on the metabolomic analysis. 
675 Each point represents the faecal metabolites in a single sample, and the mean and SEM were 676 indicated by horizontal lines. One-way ANOVA followed by Tukey's multiple comparisons 677 test was used to determine statistical significance, and the $p$ value was obtained by comparing 678 the mean of each group with the mean of the Con group, ns means no significant difference, 


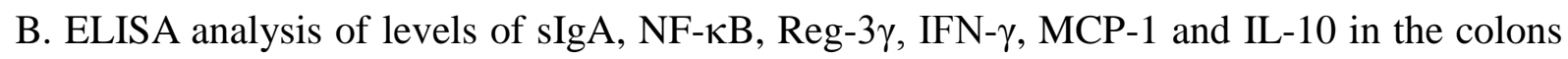
of mice from Con, Amp, NaR, LacG, LacC and LacP groups. One-way ANOVA followed by

699 Tukey's multiple comparisons test was used to determine statistical significance, and the $p$ 700 value was obtained by comparing the mean of each group with the mean of the Con group, ns 701 means no significant difference, ${ }^{*} p<0.05$, ${ }^{* *} p<0.01,{ }^{* * *} p<0.001$, ${ }^{* * * *} p<0.0001$. Values 702 are represented as mean \pm SEM of 5-8 mice per group. 
703 Table 1. Animal experimental design

\begin{tabular}{|c|c|c|}
\hline Groups & $\begin{array}{l}\text { Antibiotic treatment } \\
\text { ( } 2 \text { weeks) }\end{array}$ & $\begin{array}{l}\text { Lactobacillus therapy assay } \\
\text { (4 weeks) }\end{array}$ \\
\hline Control (Con) & $0.9 \%$ saline solution & $0.9 \%$ saline solution or sacrifice \\
\hline Ampicillin (Amp) & Ampicillin (500 mg kg-1) & Sacrifice \\
\hline Natural Restoration (NaR) & Ampicillin (500 mg kg-1) & $0.9 \%$ saline solution \\
\hline L. rhamnosus GG (LacG) & Ampicillin (500 mg kg-1) & LacG $10^{9} \mathrm{CFU}$ in $0.9 \%$ saline \\
\hline L. casei CGMCC 12435 (LacC) & Ampicillin (500 mg kg ${ }^{-1}$ ) & LacC $10^{9} \mathrm{CFU}$ in $0.9 \%$ saline \\
\hline L. plantarum CGMCC12436 (LacP) & Ampicillin (500 mg kg ${ }^{-1}$ ) & LacP $10^{9} \mathrm{CFU}$ in $0.9 \%$ saline \\
\hline
\end{tabular}


A

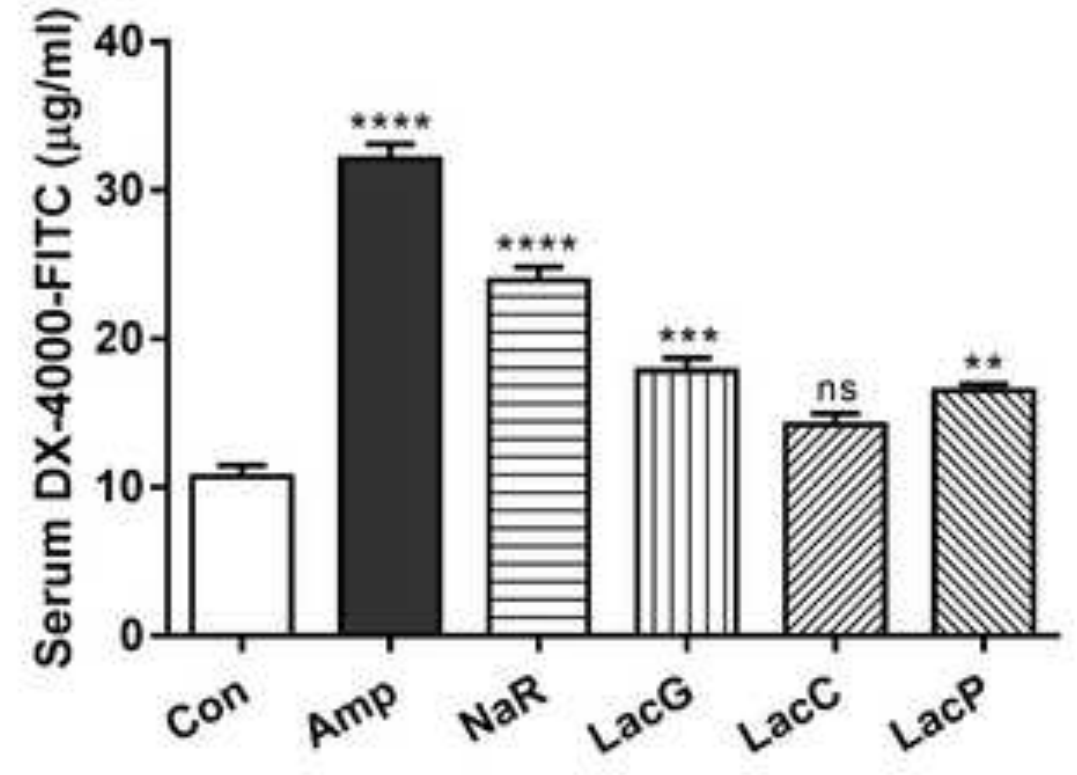

C

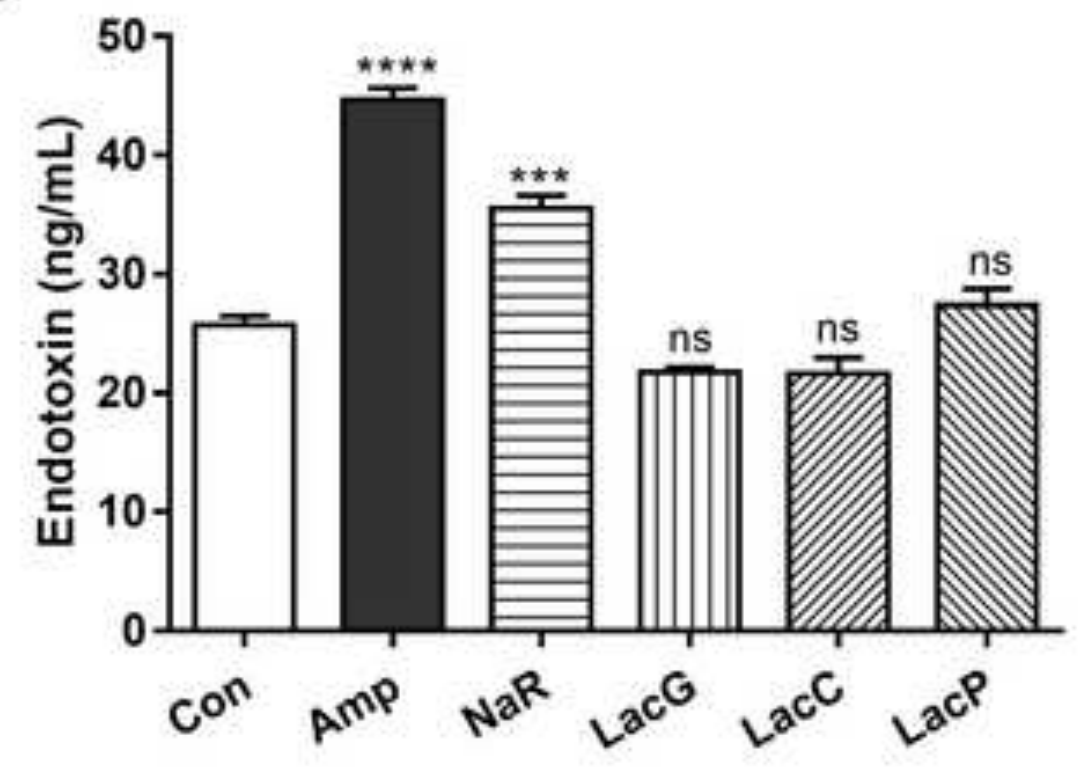

B

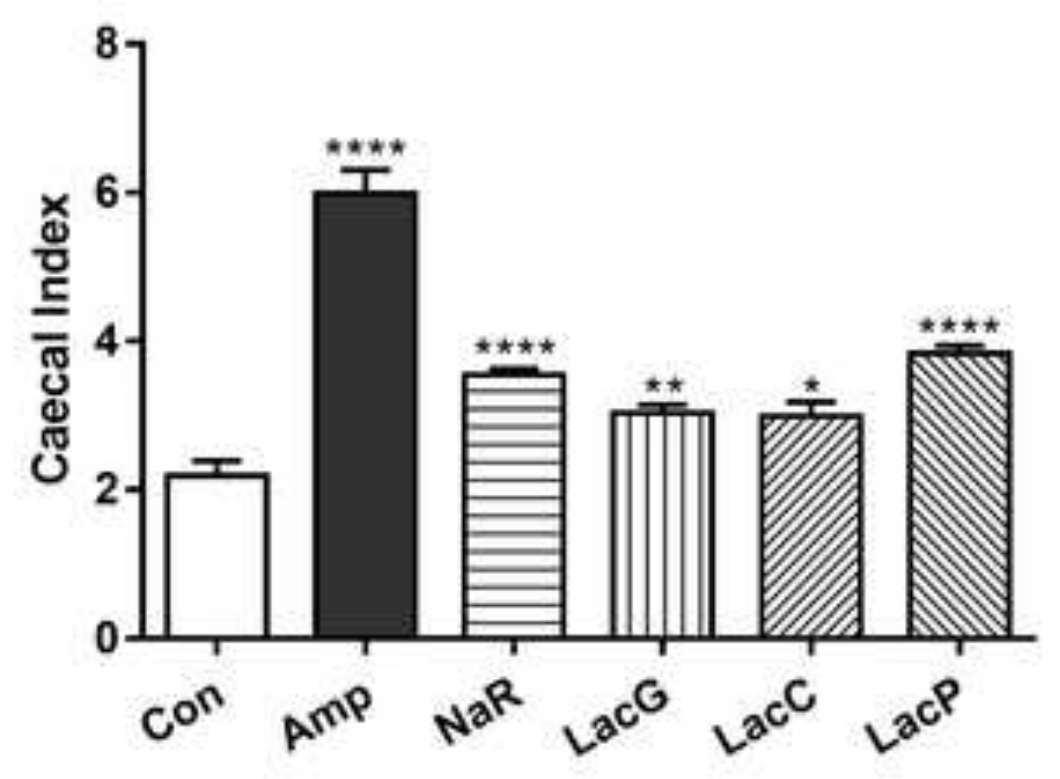

D

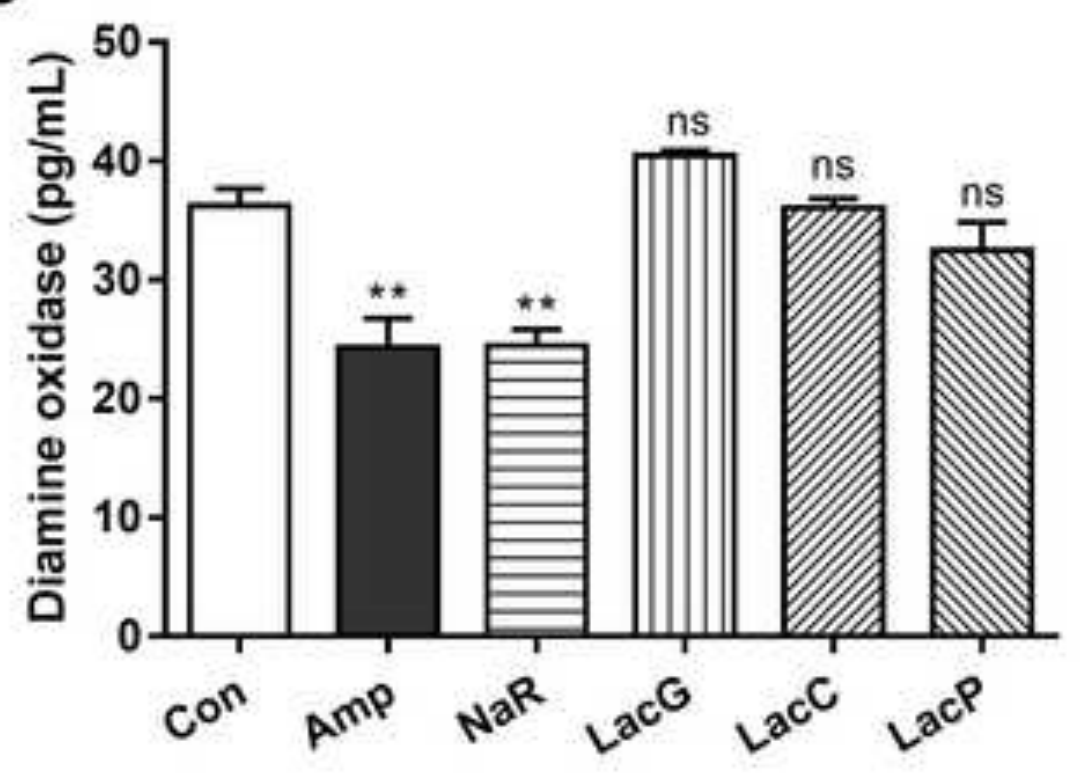




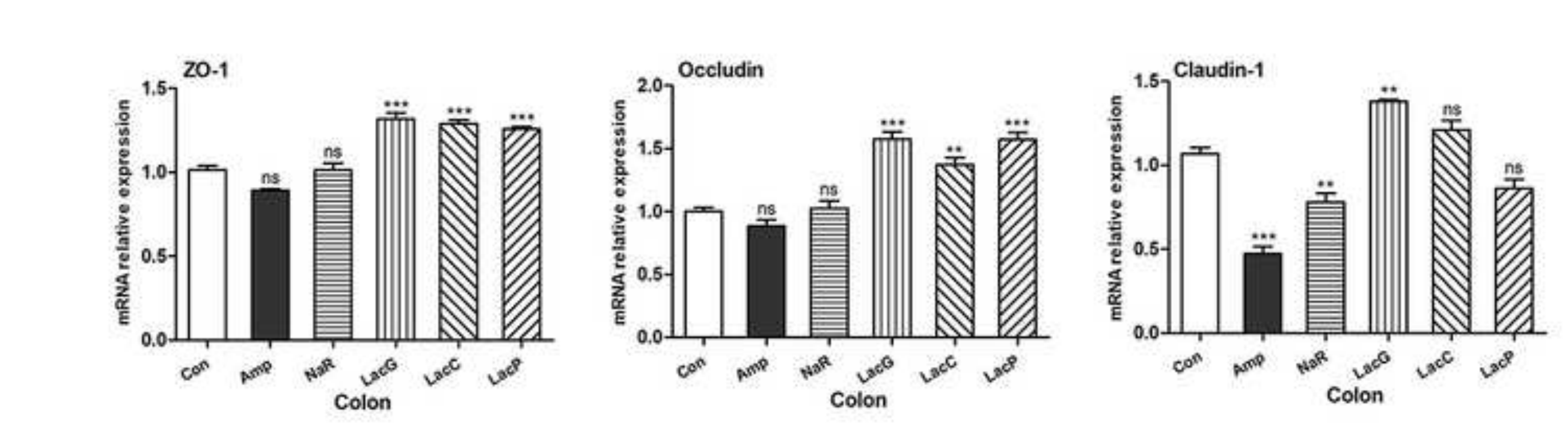

$$
\begin{aligned}
& \text { 男 }
\end{aligned}
$$


Click here to download high resolution image

A

Shannon

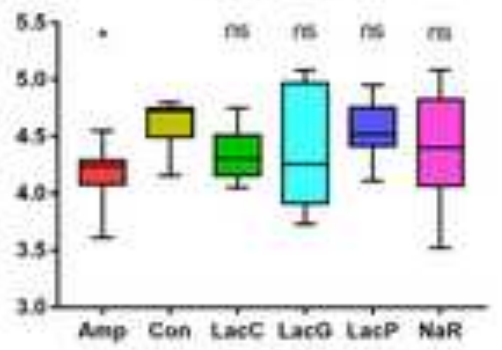

Simpson

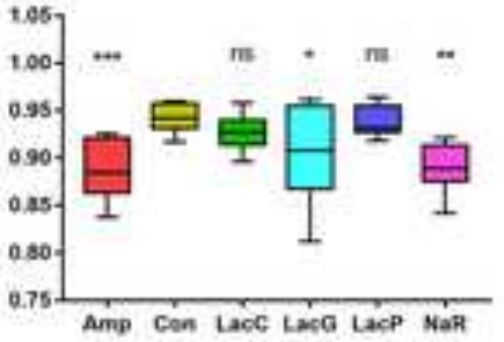

IrvSimpsen
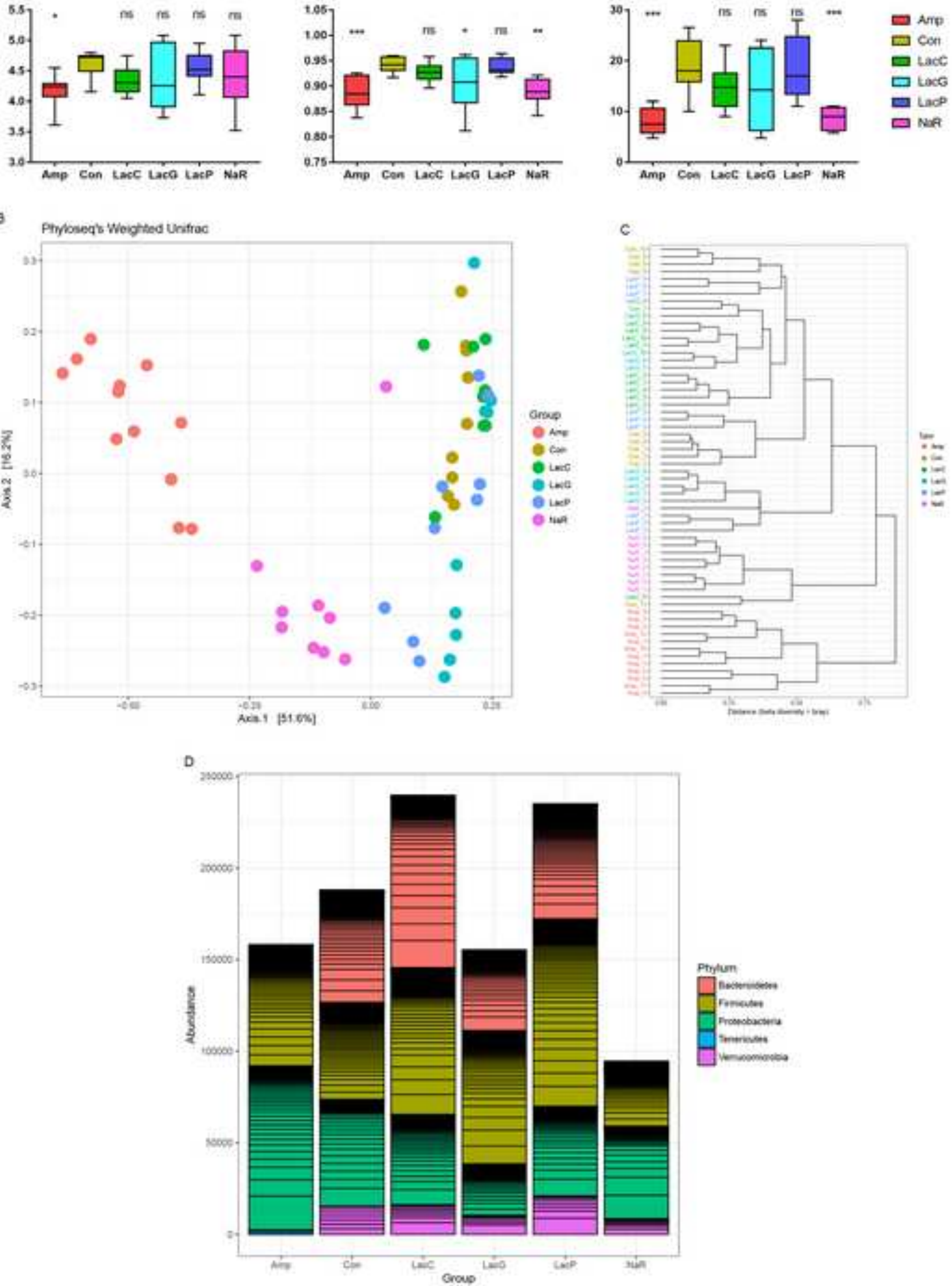


\section{Figure 4}

Click here to download high resolution image
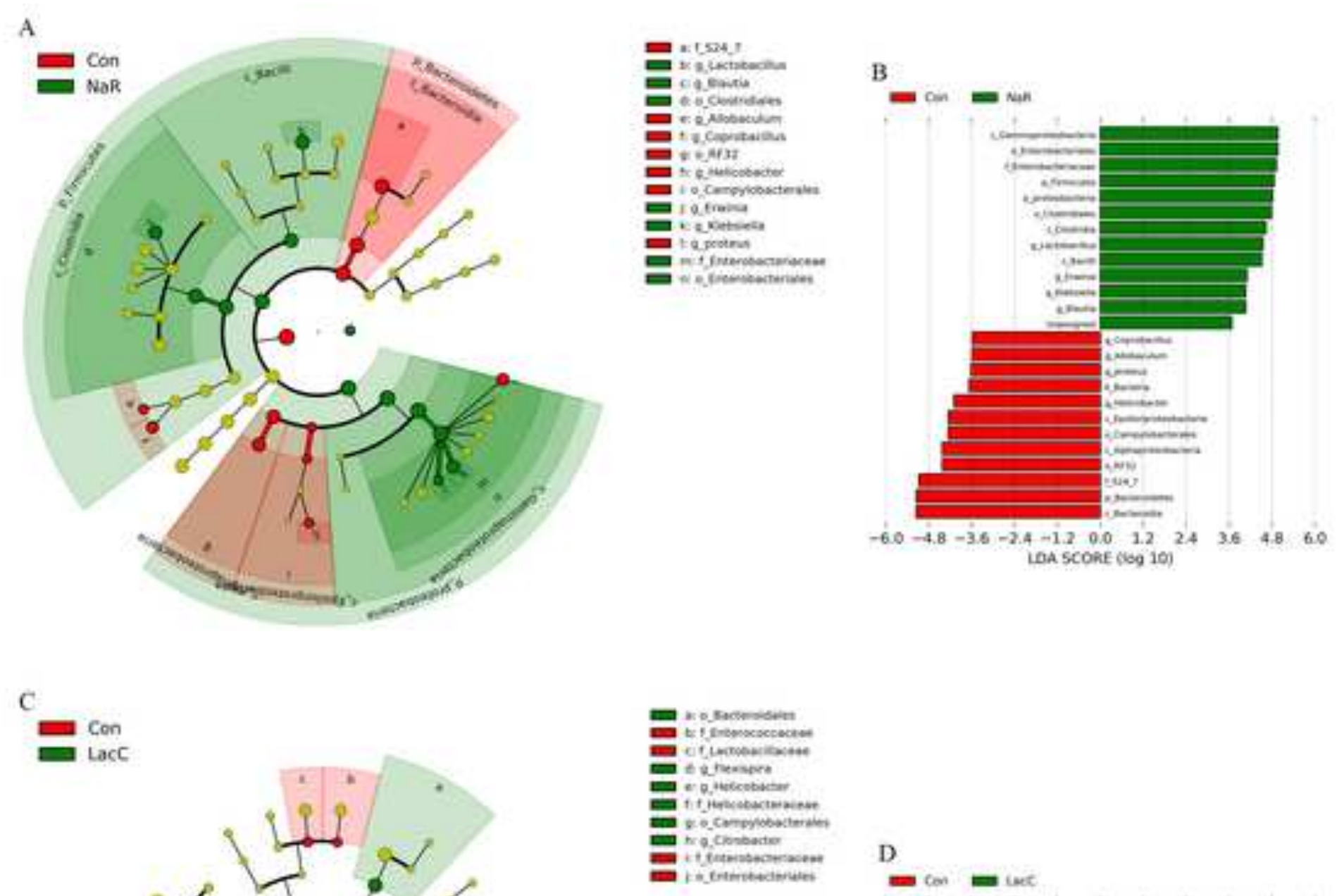

D
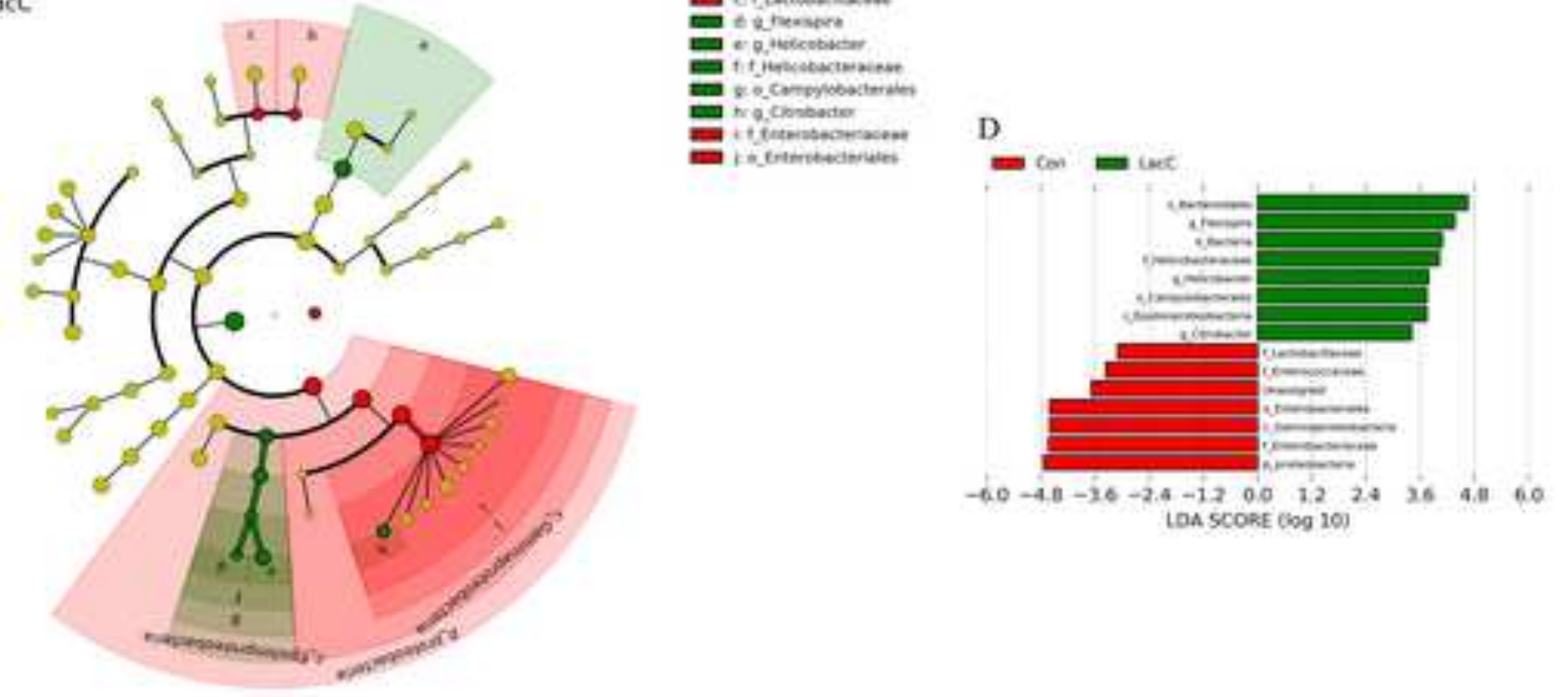

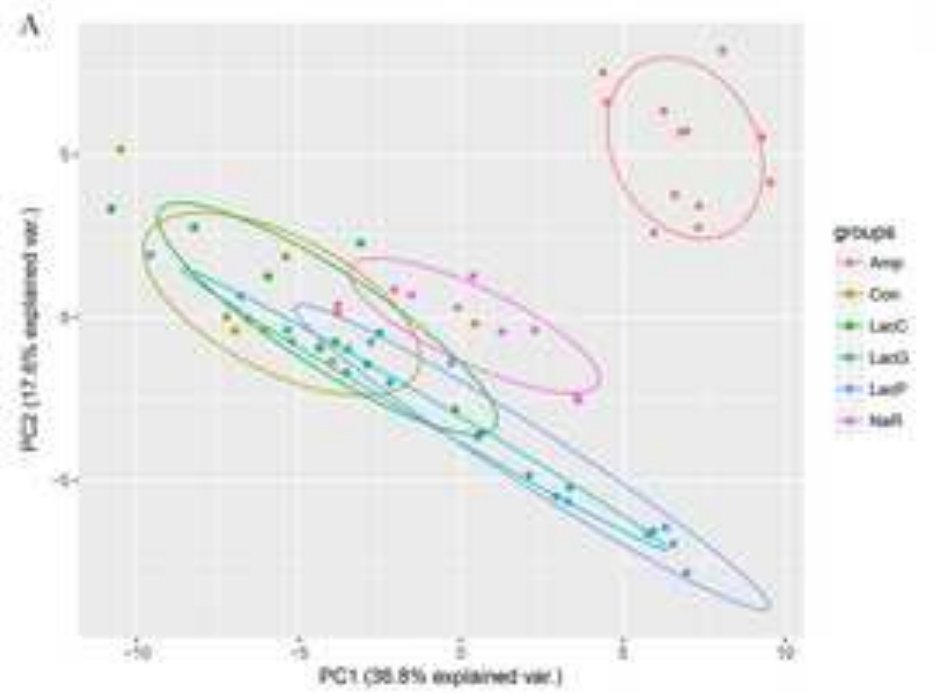

Is
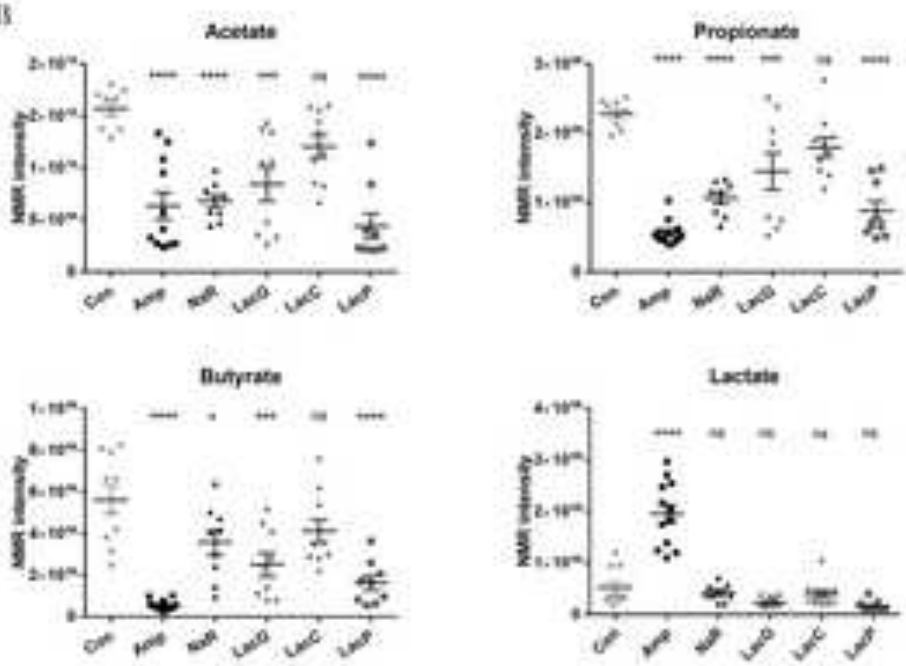

c
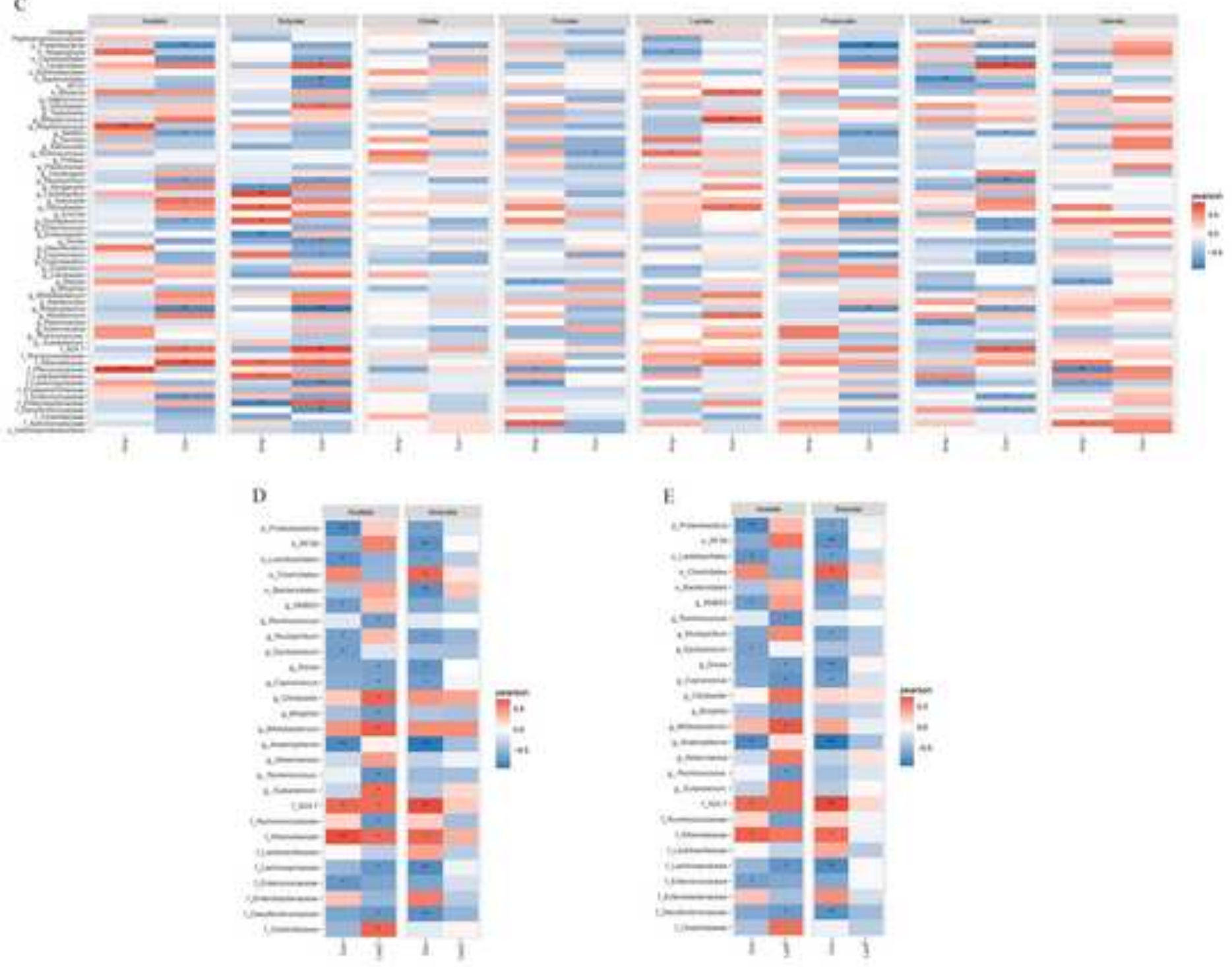

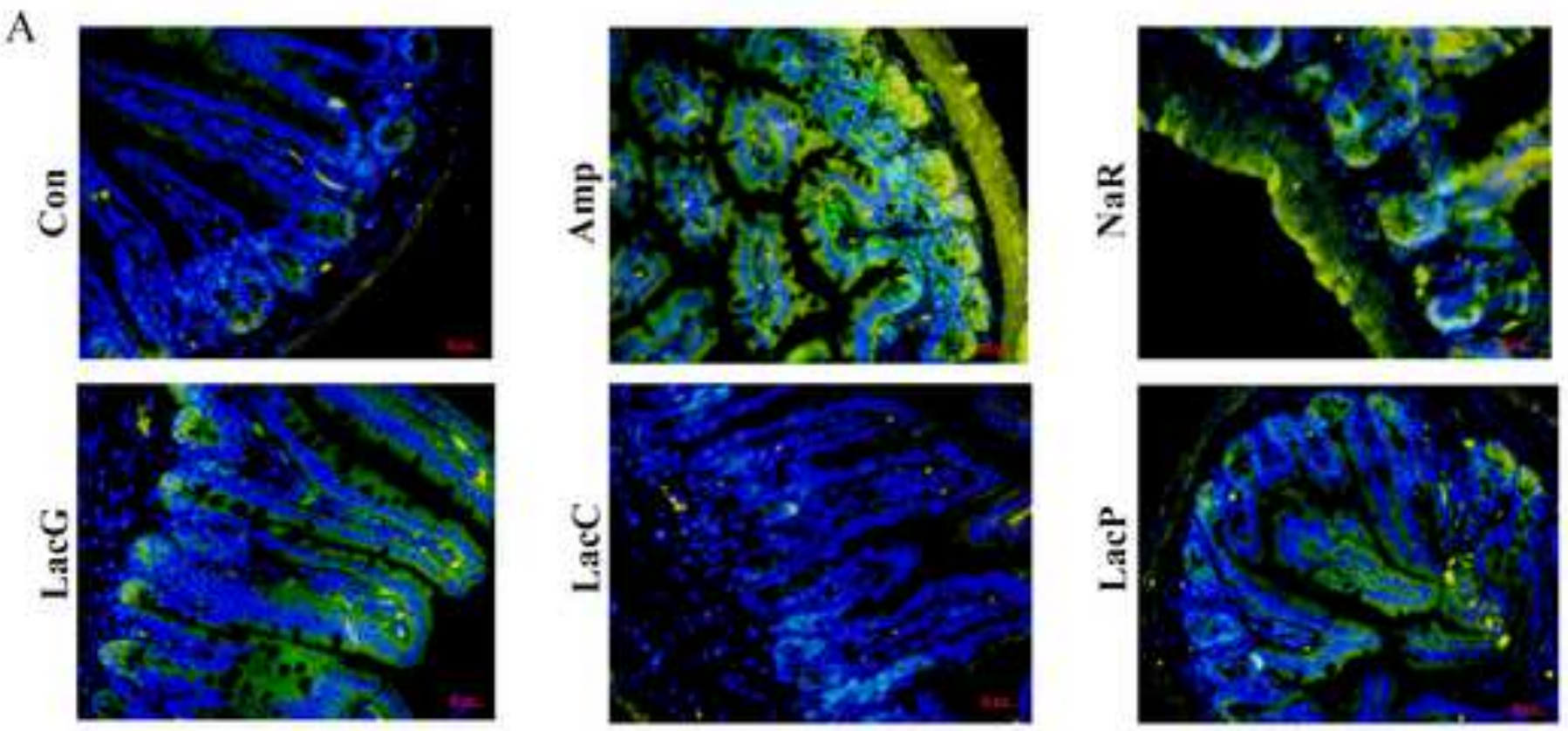

B
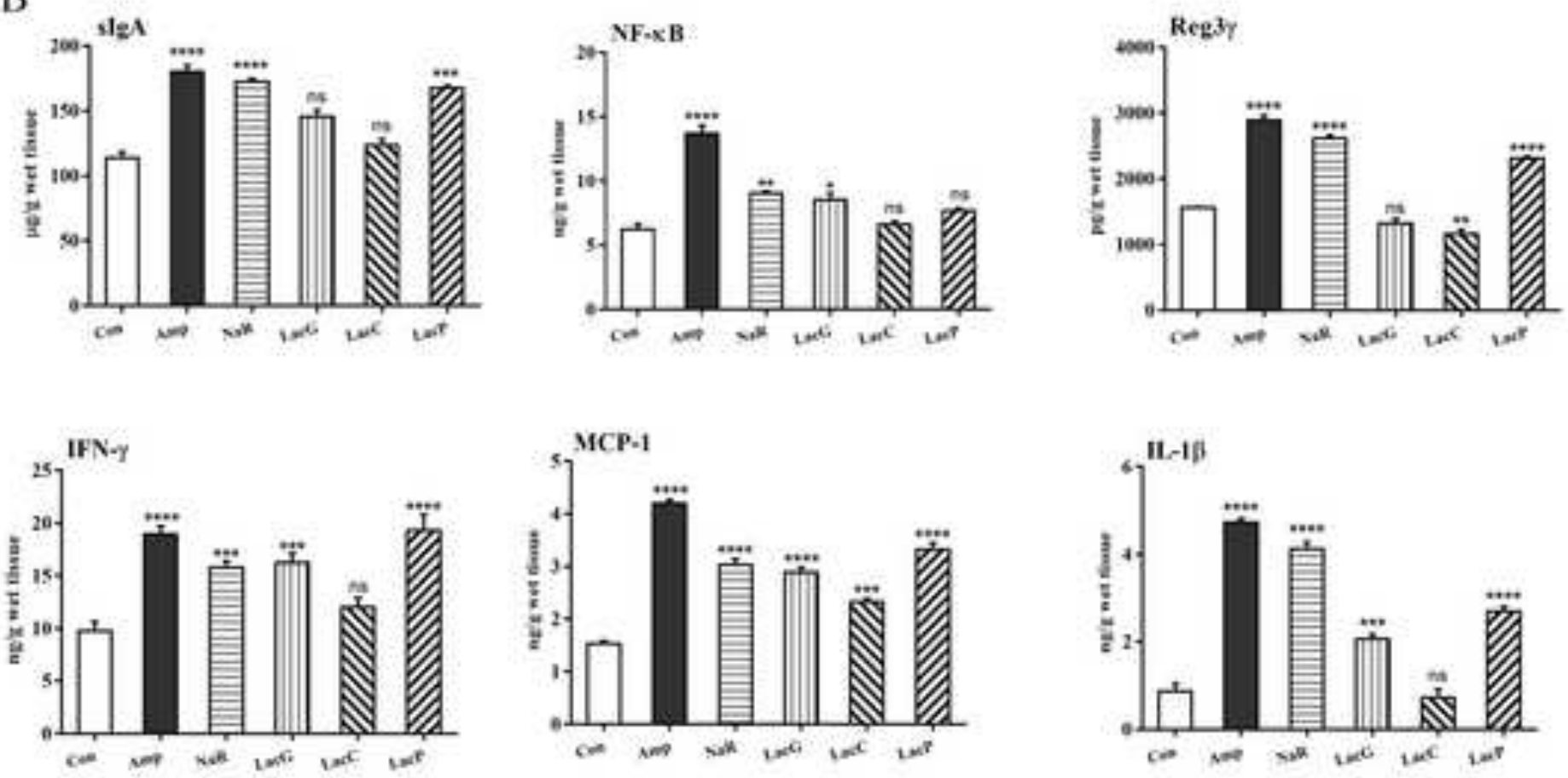


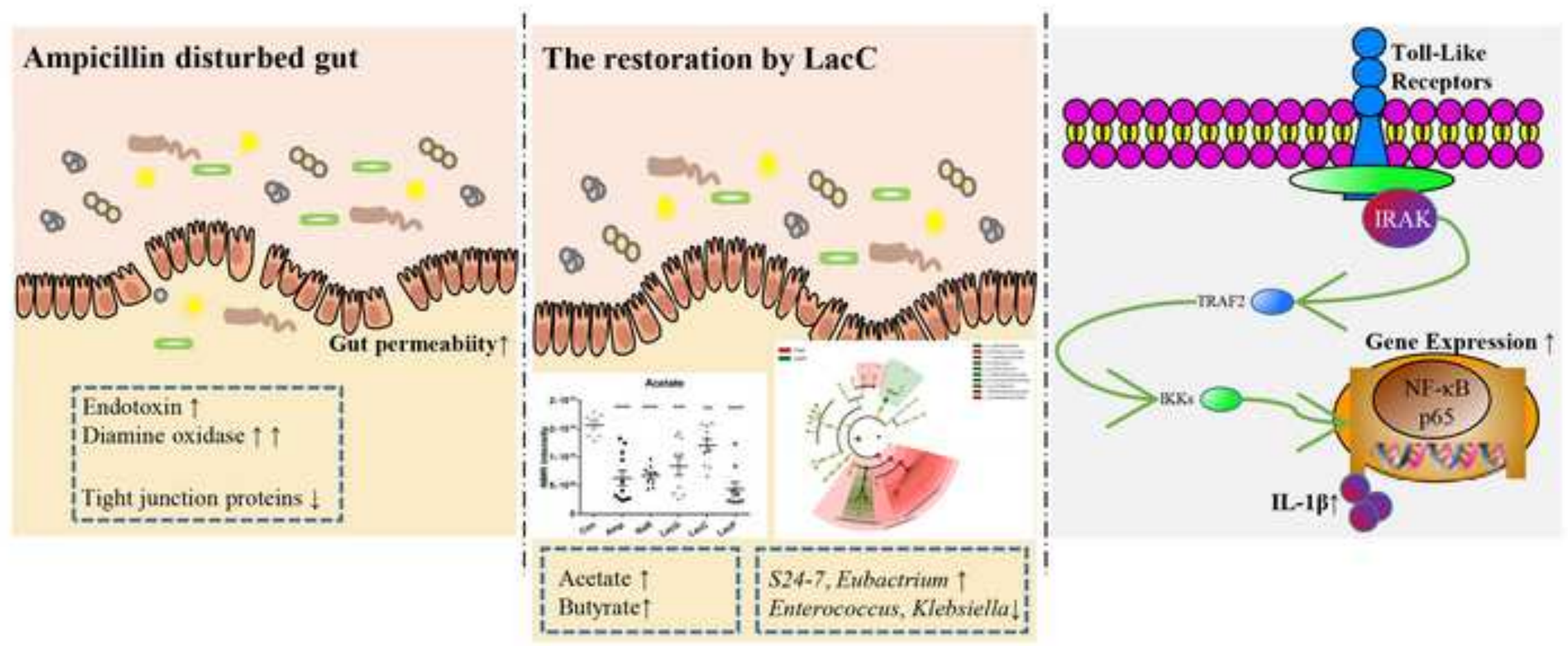


Click here to download Data in Brief: Supplementary-14Sep2018.docx

Supplementary files
Click here to downlo

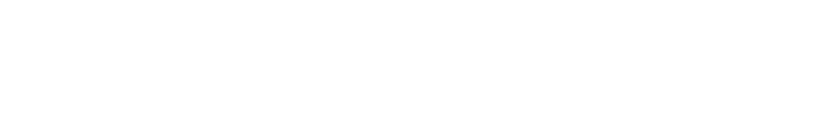
. (1) (1) (1) (1) (1) (1) (1) . . . . . . . . . . .

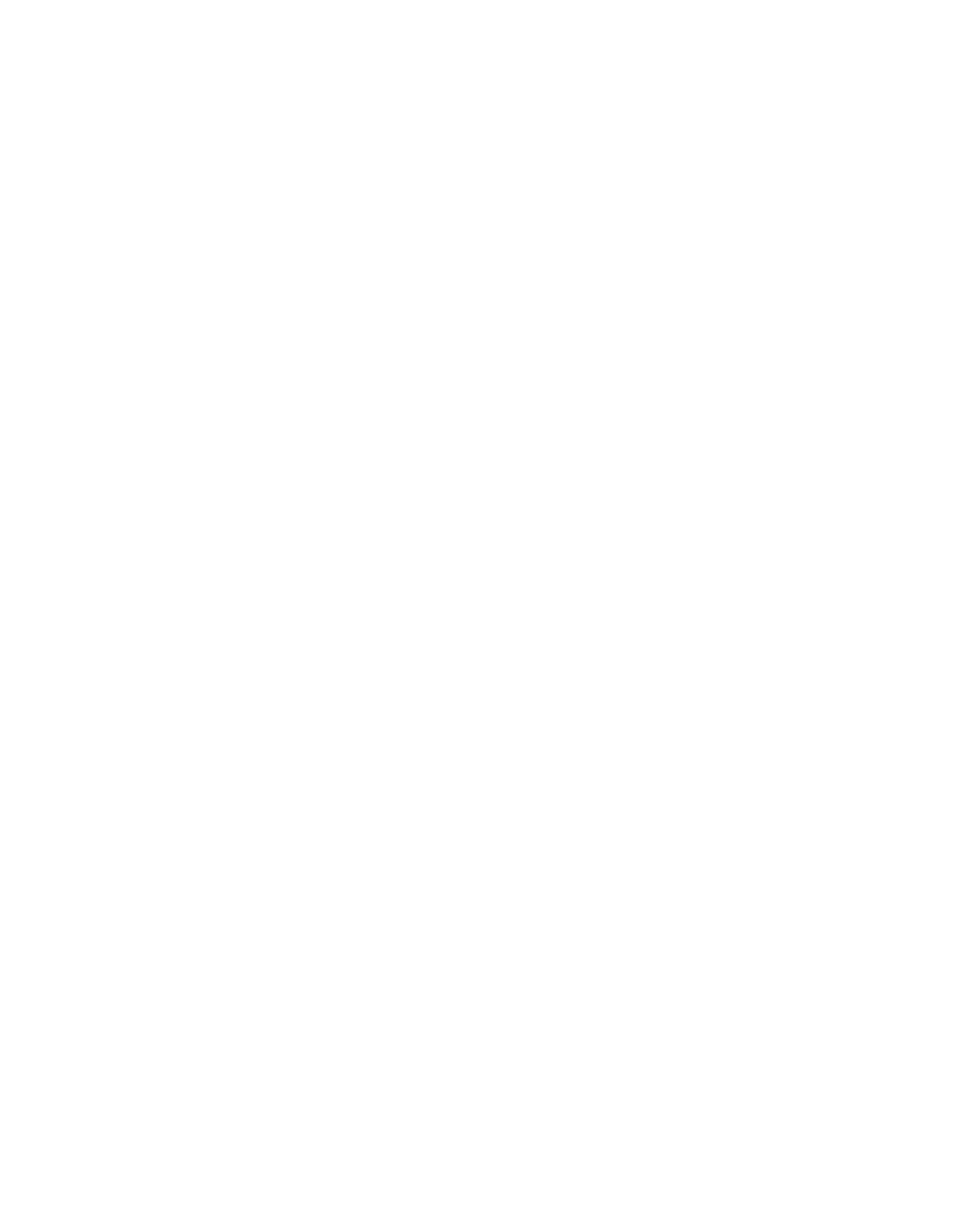




\section{Click here to download Data in Brief: MS-12sep2018_LK28092018_AN.docx}



Supplementary files
Click here to download Data in Brief: MS-12Sep2018-TrackChangesLK.docx

Click here to download Data in Brief: MS-12Sep2018-TrackChangesLK.docx

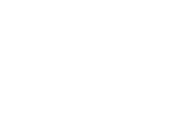
(1)

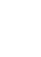
(1) (1) (1) (1) (1) (1) . . . . . . . . . . . 

Supplementary files
Click here to download Data in Brief: MS-12Sep2018-TrackChanges1.docx (1)

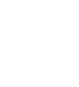

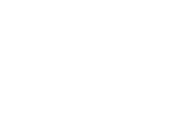

$\sqrt{3}$

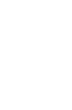

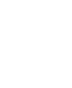
. . . . . .

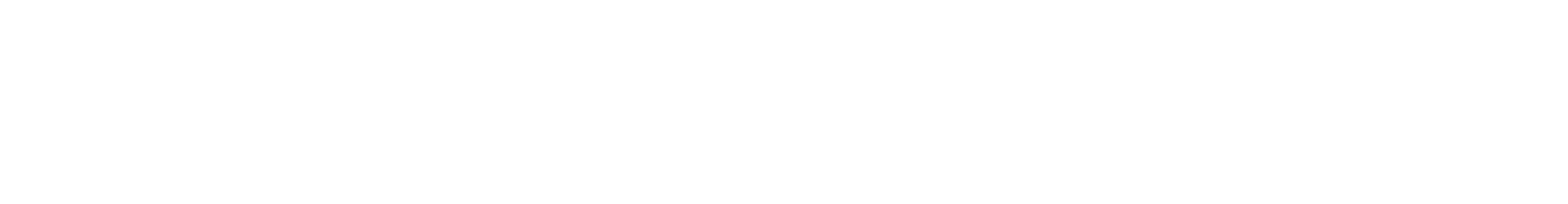


${ }^{*}$ Conflict of Interest

Declarations of interest: none. 


\section{Ethnic statement}

The entire experiment was approved by the Ethics Committee of Jiangnan University in China (JN No. 20160608-20160831/47), and the procedures were carried out according to the European Community guidelines (Directive 2010/63/EU) for the care and use of experimental animals 